\title{
Charmonium spectrum and their electromagnetic transitions with higher multipole contributions
}

\author{
Wei-Jun Deng, Hui Liu, Long-Cheng Gui *, and Xian-Hui Zhong ${ }^{\dagger}$ \\ 1) Department of Physics, Hunan Normal University, and Key Laboratory of Low-Dimensional \\ Quantum Structures and Quantum Control of Ministry of Education, Changsha 410081, China and \\ 2) Synergetic Innovation Center for Quantum Effects and Applications (SICQEA), Changsha 410081, China
}

\begin{abstract}
The charmonium spectrum is calculated with two nonrelativistic quark models, the linear potential model and the screened potential model. Using the obtained wavefunctions, we evaluate the electromagnetic transitions of charmonium states up to $4 S$ multiplet. The higher multipole contributions are included by a multipole expansion of the electromagnetic interactions. Our results are in reasonable agreement with the measurements. As conventional charmonium states, the radiative decay properties of the newly observed charmonium-like states, such as $X(3823), X(3872), X(4140 / 4274)$, are discussed. The $X(3823)$ as $\psi_{2}(1 D)$, its radiative decay properties well agree with the observations. From the radiative decay properties of $X(3872)$, one can not exclude it as a $\chi_{c 1}(2 P)$ dominant state. We also give discussions of possibly observing the missing charmonium states in radiative transitions, which might provide some useful references to look for them in forthcoming experiments. The higher multipole contributions to the electromagnetic transitions are analyzed as well. It is found that the higher contribution from the magnetic part could give notable corrections to some E1 dominant processes by interfering with the E1 amplitudes. Our predictions for the normalized magnetic quadrupole amplitudes $M_{2}$ of the $\chi_{c 1,2}(1 P) \rightarrow J / \psi \gamma$ processes are in good agreement with the recent CLEO measurements.
\end{abstract}

PACS numbers: 12.39.Jh, 12.39.Pn, 13.20.Gd, 14.40.Pq

\section{INTRODUCTION}

During the past few years, great progress has been achieved in the observation of the charmonia [1-5]. From the review of the Particle Data Group (PDG) [6], one can see that many new charmonium-like " $X Y Z$ " states above open-charm thresholds states have been observed at Belle, BaBar, LHC, BESIII, CLEO and so on. The observations of these new states not only deepen our understanding of the charmonium physics, but also bring us many mysteries in this field to be uncovered [3-5]. If these newly observed " $X Y Z$ " states, such as $X(3872), X(3915), X(4140 / 4274)$ and $Y(4260)$, are assigned as conventional charmonium states, some properties, such as measured mass and decay modes may be inconsistent with the predictions. Thus, how to identify these newly observed charmonium-like " $X Y Z$ " states and how to understand their uncommon nature are great challenges for physicist.

Stimulated by the extensive progress made in the observation of the charmonia, in this work we study the mass spectrum and electromagnetic (EM) transitions of charmonium within the widely used linear potential model [7-9], and the screened potential model [10, 11]. As we know, the EM decays of a hadron are sensitive to its inner structure. The study of the EM decays not only is crucial for us to determine the quantum numbers of the newly observed charmonium states, but also provides very useful references for our search for the missing charmonium states in experiments. To study the charmonium spectrum and/or their EM decays, beside the widely used potential models [7-18], some other models, such as lattice QCD [19-26], QCD sum rules [27-29], coupled-channel quark models [30], effective Lagrangian approach [31, 32],

\footnotetext{
*E-mail: guilongcheng@ihep.ac.cn

${ }^{\dagger}$ E-mail: zhongxh@hunnu.edu.cn
}

nonrelativistic effective field theories of QCD [33-36], relativistic quark model [37], relativistic Salpeter method [38], light front quark model [39], Coulomb gauge approach [40], and generalized screened potential model [41] have been employed in theory. Recently, the hadronic loop contributions to the radiative decay of charmonium states were also discussed in Refs. [42-44]. Although there are many studies about the EM decays of charmonium states, many properties are not well understood. For example the predictions for the $\chi_{c J}(1 P) \rightarrow J / \psi \gamma$ and $\psi(3770) \rightarrow \chi_{c J}(1 P) \gamma$ processes are rather different in various models [45]. These differences may come from the wavefunctions of charmonium states adopted, the higher EM multipole amplitude contributions, the coupled-channel effects and so on. Thus, to clarify these puzzles, more studies are needed.

In this work, we mainly focus on the following issues: (i) To clearly show the model dependence of the higher charmonium states, we calculate the charmonium spectroscopy within two typical models, i.e., linear and screened potential models. As done in the literature, e.g. [7-9, 18], the spindependent potentials are dealt with non-perturbatively so that the corrections of the spin-dependent interactions to the wavefunctions can be included. (ii) We further analyze the EM transitions between charmonium states. Based on the obtained radiative decay properties and mass spectrum, we discuss the classifications of the newly observed charmonium-like states; while for the missing excited states we suggest strategies to find them in radiative transitions. (iii) Finally, we discuss the possible higher EM multipole contributions to a EM transition process.

The paper is organized as follows. In Sec. II the charmonium spectroscopy is calculated within both the linear and screened potential models. In Sec. IIII firstly we give an introduction of EM transitions described in present work. Then, using the wavefunctions obtained from both the linear and screened potential models, we analyze the EM decays of char- 
monium states. Finally, a summary is given in Sec. IV

TABLE I: Charmonium mass spectrum. LP and SP stand for our calculated masses with the linear potential and screened potential models, respectively. For comparison, the measured masses $(\mathrm{MeV})$ from the PDG [6], and the previous predictions with screened potential in Ref. [11] and linear potential in Ref. [8] are also listed.

\begin{tabular}{|c|c|c|c|c|c|c|c|}
\hline$n^{2 S+1} L_{J}$ & name & $J^{P C}$ & Exp. [6] & [8] & [11] & LP & SP \\
\hline $1^{3} S_{1}$ & $J / \psi$ & $1^{--}$ & $3097^{a}$ & 3090 & 3097 & 3097 & 3097 \\
\hline $1^{1} S_{0}$ & $\eta_{c}(1 S)$ & $0^{-+}$ & $2984^{a}$ & 2982 & 2979 & 2983 & 2984 \\
\hline $2^{3} S_{1}$ & $\psi(2 S)$ & $1^{--}$ & $3686^{a}$ & 3672 & 3673 & 3679 & 3679 \\
\hline $2^{1} S_{0}$ & $\eta_{c}(2 S)$ & $0^{-+}$ & $3639^{a}$ & 3630 & 3623 & 3635 & 3637 \\
\hline $3^{3} S_{1}$ & $\psi(3 S)$ & $1^{--}$ & $4040^{a}$ & 4072 & 4022 & 4078 & 4030 \\
\hline $3^{1} S_{0}$ & $\eta_{c}(3 S)$ & $0^{-+}$ & & 4043 & 3991 & 4048 & 4004 \\
\hline $4^{3} S_{1}$ & $\psi(4 S)$ & $1^{--}$ & $4415 ?$ & 4406 & 4273 & 4412 & 4281 \\
\hline $4^{1} S_{0}$ & $\eta_{c}(4 S)$ & $0^{-+}$ & & 4384 & 4250 & 4388 & 4264 \\
\hline $5^{3} S_{1}$ & $\psi(5 S)$ & $1^{--}$ & & & 4463 & 4711 & 4472 \\
\hline $5^{1} S_{0}$ & $\eta_{c}(5 S)$ & $0^{-+}$ & & & 4446 & 4690 & 4459 \\
\hline $1^{3} P_{2}$ & $\chi_{c 2}(1 P)$ & $2^{++}$ & $3556^{a}$ & 3556 & 3554 & 3552 & 3553 \\
\hline $1^{3} P_{1}$ & $\chi_{c 1}(1 P)$ & $1^{++}$ & $3511^{a}$ & 3505 & 3510 & 3516 & 3521 \\
\hline $1^{3} P_{0}$ & $\chi_{c 0}(1 P)$ & $0^{++}$ & $3415^{a}$ & 3424 & 3433 & 3415 & 3415 \\
\hline $1^{1} P_{1}$ & $h_{c}(1 P)$ & $1^{+-}$ & $3525^{a}$ & 3516 & 3519 & 3522 & 3526 \\
\hline $2^{3} P_{2}$ & $\chi_{c 2}(2 P)$ & $2^{++}$ & $3927^{a}$ & 3972 & 3937 & 3967 & 3937 \\
\hline $2^{3} P_{1}$ & $\chi_{c 1}(2 P)$ & $1^{++}$ & & 3925 & 3901 & 3937 & 3914 \\
\hline $2^{3} P_{0}$ & $\chi_{c 0}(2 P)$ & $0^{++}$ & $3918 ?$ & 3852 & 3842 & 3869 & 3848 \\
\hline $2^{1} P_{1}$ & $h_{c}(2 P)$ & $1^{+-}$ & & 3934 & 3908 & 3940 & 3916 \\
\hline $3^{3} P_{2}$ & $\chi_{c 2}(3 P)$ & $2^{++}$ & & 4317 & 4208 & 4310 & 4211 \\
\hline $3^{3} P_{1}$ & $\chi_{c 1}(3 P)$ & $1^{++}$ & & 4271 & 4178 & 4284 & 4192 \\
\hline $3^{3} P_{0}$ & $\chi_{c 0}(3 P)$ & $0^{++}$ & & 4202 & 4131 & 4230 & 4146 \\
\hline $3^{1} P_{1}$ & $h_{c}(3 P)$ & $1^{+-}$ & & 4279 & 4184 & 4285 & 4193 \\
\hline $1^{3} D_{3}$ & $\psi_{3}(1 D)$ & $3^{--}$ & & 3806 & 3799 & 3811 & 3808 \\
\hline $1^{3} D_{2}$ & $\psi_{2}(1 D)$ & $2^{--}$ & $3823^{a}$ & 3800 & 3798 & 3807 & 3807 \\
\hline $1^{3} D_{1}$ & $\psi_{1}(1 D)$ & $1^{--}$ & $3778^{a}$ & 3785 & 3787 & 3787 & 3792 \\
\hline $1^{1} D_{2}$ & $\eta_{c 2}(1 D)$ & $2^{-+}$ & & 3799 & 3796 & 3806 & 3805 \\
\hline $2^{3} D_{3}$ & $\psi_{3}(2 D)$ & $3^{--}$ & & 4167 & 4103 & 4172 & 4112 \\
\hline $2^{3} D_{2}$ & $\psi_{2}(2 D)$ & $2^{--}$ & & 4158 & 4100 & 4165 & 4109 \\
\hline $2^{3} D_{1}$ & $\psi_{1}(2 D)$ & $1^{--}$ & $4191 ?$ & 4142 & 4089 & 4144 & 4095 \\
\hline $2^{1} D_{2}$ & $\eta_{c 2}(2 D)$ & $2^{-+}$ & & 4158 & 4099 & 4164 & 4108 \\
\hline $3^{3} D_{3}$ & $\psi_{3}(3 D)$ & $3^{--}$ & & & 4331 & 4486 & 4340 \\
\hline $3^{3} D_{2}$ & $\psi_{2}(3 D)$ & $2^{--}$ & & & 4327 & 4478 & 4337 \\
\hline $3^{3} D_{1}$ & $\psi_{1}(3 D)$ & $1^{--}$ & & & 4317 & 4456 & 4324 \\
\hline $3^{1} D_{2}$ & $\eta_{c 2}(3 D)$ & $2^{-+}$ & & & 4326 & 4478 & 4336 \\
\hline
\end{tabular}

${ }^{a}$ These masses for the 12 well-esbalished $c \bar{c}$ states are used as input to determine the model parameters.

\section{MASS SPECTROSCOPY}

\section{A. FORMALISM}

In this work, the mass and space wavefunction of a charmonium state are determined by the Schrödinger equation with a conventional quarkonium potential. The effective potential of spin-independent term $V(r)$ between the quark and antiquark is regarded as the sum of Lorentz vector $V_{V}(r)$ and Lorentz scalar $V_{s}(r)$ contributions [1], i.e.,

$$
V(r)=V_{V}(r)+V_{s}(r) .
$$

The Lorentz vector potential $V_{V}(r)$ is adopted the standard color Coulomb form:

$$
V_{V}(r)=-\frac{4}{3} \frac{\alpha_{s}}{r}
$$

The Lorentz scalar $V_{s}(r)$ might be taken as

$$
V_{s}(r)=\left\{\begin{array}{ll}
b r, & \text { linear potential } \\
\frac{b}{\mu}\left(1-e^{-\mu r}\right), & \text { screened potential }
\end{array},\right.
$$

where $r$ is the distance between the quark and antiquark. The linear potential $b r$ is widely used in the potential models. Considering the screening effect from the vacuum polarization effect of the dynamical light quark might soft the linear potential at large distances [46, 47], people suggested a screened potential $b\left(1-e^{-\mu r}\right) / \mu$ in the calculations as well $[10,11,15,16,48]$. Here $\mu$ is the screening factor which makes the long-range scalar potential of $V_{s}(r)$ behave like $b r$ when $r \ll 1 / \mu$, and becomes a constant $b / \mu$ when $r \gg 1 / \mu$. The main effects of the screened potential on the spectrum is that the masses of the higher excited states are lowered.

Following the method in Refs. [8, 11], we include three spin-dependent potentials in our calculations. For the spinspin contact hyperfine potential, we take the Gaussiansmeared form [8]

$$
H_{S S}=\frac{32 \pi \alpha_{s}}{9 m_{c}^{2}} \tilde{\delta}_{\sigma}(r) \mathbf{S}_{c} \cdot \mathbf{S}_{\bar{c}},
$$

where $\mathbf{S}_{c}$ and $\mathbf{S}_{\bar{c}}$ are spin matrices acting on the spins of the quark and antiquark. We take $\tilde{\delta}_{\sigma}(r)=(\sigma / \sqrt{\pi})^{3} e^{-\sigma^{2} r^{2}}$ as in Ref. [8]. The five parameters in the above equations $\left(\alpha_{s}, b, \mu\right.$, $\left.m_{c}, \sigma\right)$ are determined by fitting the spectrum.

For the spin-orbit term and the tensor term, we take the common forms obtained from the leading-order perturbation theory:

$$
H_{S L}=\frac{1}{2 m_{c}^{2} r}\left(3 \frac{d V_{V}}{d r}-\frac{d V_{s}}{d r}\right) \mathbf{L} \cdot \mathbf{S}
$$

and

$$
H_{T}=\frac{1}{12 m_{c}^{2}}\left(\frac{1}{r} \frac{d V_{V}}{d r}-\frac{d^{2} V_{V}}{d r^{2}}\right) S_{T},
$$

where $\mathbf{L}$ is the relative orbital angular momentum of $c$ and $\bar{c}$ quarks, $\mathbf{S}=\mathbf{S}_{c}+\mathbf{S}_{\bar{c}}$ is the total quark spin, and the spin tensor $S_{T}$ is defined by

$$
S_{T}=6 \frac{\mathbf{S} \cdot \mathbf{r S} \cdot \mathbf{r}}{r^{2}}-2 \mathbf{S}^{2}
$$

By solving the radial Schrödinger equation $\frac{d^{2} u(r)}{d r^{2}}+$ $2 \mu_{R}\left[E-V_{c \bar{c}}(r)-\frac{L(L+1)}{2 \mu_{R} r^{2}}\right] u(r)=0$, with $V_{c \bar{c}}(r) \equiv V(r)+H_{S S}+$ 

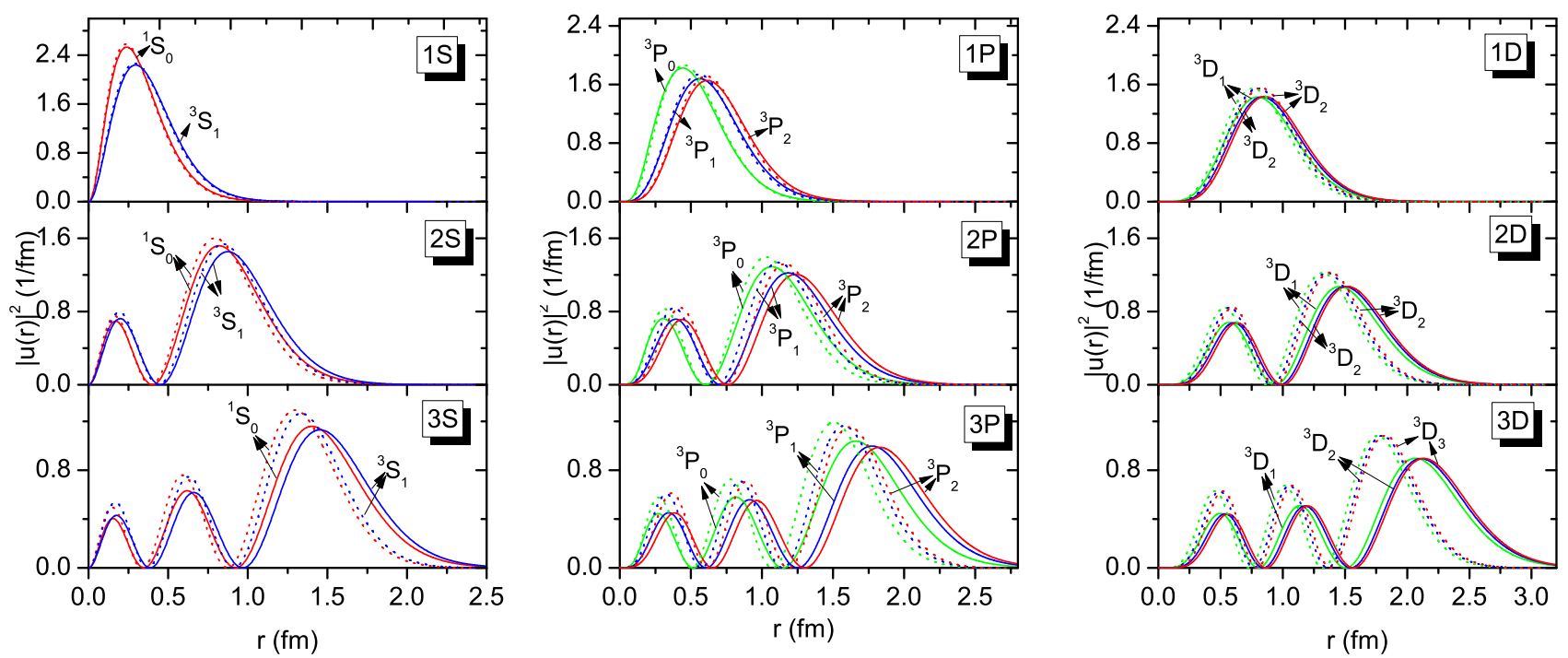

FIG. 1: (Color online) Predicted radial probability density $|u(r)|^{2}$ for $S$-, $P$ - and $D$-wave charmonium states up to $n=3$ shell. The dotted and solid curves stand for the results obtained from the linear and screened potential models, respectively.

$H_{S L}+H_{T}$ and $\mu_{R} \equiv m_{c} m_{\bar{c}} /\left(m_{c}+m_{\bar{c}}\right)$, we obtain the wavefunction $u(r)$ and the mass $M_{c \bar{c}}=2 m_{c}+E$ for a charmonium state. For simplification, the spin-dependent interactions can be dealt with perturbatively. Although the meson mass obtains perturbative corrections from these spin-dependent potentials, the wave functions obtain no corrections from them. Thus, to reasonably include the corrections from these spindependent potentials to both the mass and wave function of a meson state, we deal with the spin-dependent interactions nonperturbatively.

In this work, we solve the radial Schrödinger equation by using the three-point difference central method from central $(r=0)$ towards outside $(r \rightarrow \infty)$ point by point. The details of this method can be found in Ref. [49]. To overcome the singular behavior of $1 / r^{3}$ in the spin-dependent potentials, following the method of our previous work [50], we introduce a cutoff distance $r_{c}$ in the calculation. Within a small range $r \in\left(0, r_{c}\right)$, we let $1 / r^{3}=1 / r_{c}^{3}$. It is found that the masses of the ${ }^{3} P_{0}$ states are sensitive to the cutoff distance $r_{c}$, which is easily determined by the mass of $\chi_{c 0}(1 P)$.

Considering the progress in the charmonium spectrum in recent years, we do not use the old parameter sets determined in Refs. [8, 11]. Combining the new measurements, we slightly adjust the parameter sets of Refs. [8, 11] to better describe the data. By fitting the masses of the 12 wellestablished $c \bar{c}$ states given in Tab. [1] we obtain the parameter sets for the linear potential model and screened potential model, which are given in Tab. III.

\section{B. Results and discussions}

Our calculated masses for the $n S(n \leq 5), n P$ and $n D$ $(n \leq 3)$ charmonium states with both the linear and screened
TABLE II: Quark model parameters determined by the 12 wellestablished $c \bar{c}$ states given in Tab.!

\begin{tabular}{c|c|c}
\hline \hline Parameter & Linear potential model & Screened potential model \\
\hline$m_{c}(\mathrm{GeV})$ & 1.4830 & 1.4110 \\
$\alpha_{s}$ & 0.5461 & 0.5070 \\
$b\left(\mathrm{GeV}^{2}\right)$ & 0.1425 & 0.2100 \\
$\sigma(\mathrm{GeV})$ & 1.1384 & 1.1600 \\
$r_{c}(\mathrm{fm})$ & 0.202 & 0.180 \\
$\mu(\mathrm{GeV})$ & $\ldots$ & 0.0979 \\
\hline \hline
\end{tabular}

potential models have been listed in Tab. I respectively. It is found the mass spectrum calculated from the three-point difference central method is consistent with the previous calculations [8, 11]. For the states with a mass of $M<4.1$ $\mathrm{GeV}$, both linear and screened potential models give a reasonable description of the mass spectrum compared with the data. However, for the higher resonances with a mass of $M>4.1$ $\mathrm{GeV}$, the predictions between these two models are very different. In the linear potential model, the well-established states $\psi(4160)$ and $\psi(4415)$ could be assigned as the $\psi_{1}(2 D)$ and $\psi(4 S)$, respectively. However, in the screened potential model, the $\psi(4415)$ might be assigned as $\psi(5 S)$ [11], while for $\psi(4160)$, the predicted mass are about $100 \mathrm{MeV}$ less than the measurements. Comparing with the linear potential model, an obvious feature of the screened potential model is that it provides a compressed mass spectrum, which permits many new charmonium-like " $X Y Z$ " states be accomodated in the conventional higher charmonium states [11]. Lately, BESIII Collaboration observed two resonant structures, one with a mass of $\sim 4222 \mathrm{MeV}$ and a width of $\sim 44 \mathrm{MeV}$, and the other with a mass of $\sim 4320 \mathrm{MeV}$ and a width of $\sim 101 \mathrm{MeV}$, in the 
cross section for the process $e^{+} e^{-} \rightarrow \pi^{+} \pi^{-} J / \psi$ [51], which may correspond to the $J^{P C}=1^{--}$states $X(4260)$ and $X(4360)$ from the PDG [6], respectively. It is found that within the screened potential model, $X(4260)$ and $X(4360)$ are good candidates of the $\psi(4 S)$ and $\psi_{1}(3 D)$, respectively. Furthermore, we should mention that recently two new charmonium-like states $X(4140)$ and $X(4274)$ were confirmed by the LHCb collaboration [52]. Their quantum numbers are determined to be $J^{P C}=1^{++}$. Within the linear potential model the $X(4274)$ might be identified as the $\chi_{c 1}(3 P)$ state. While within the screened potential model, the $X(4140)$ is a good candidate of $\chi_{c 1}(3 P)$. However, neither the linear potential model nor the screened model can give two conventional $J^{P C}=1^{++}$charmonium states with masses around 4.14 and $4.27 \mathrm{GeV}$ at the same time, which may indicate the exotic nature of $X(4140)$ and/or $X(4274)$.

Furthermore, in Tab. III] we give our predictions of the hyperfine splittings for some $S$-wave states, and fine splittings for some $P$-wave states with the linear and screened potentials, respectively. For a comparison, the world average data from the PDG [6] and the previous predictions in Refs. [8, 11] are listed in the same table as well. It is found that both the linear and screened potential models give comparable results. The predicted splittings are in agreement with the world average data [6]. It should be mentioned that both the linear and screened potential models obtain a similar fine splitting between $\chi_{c 2}(2 P)$ and $\chi_{c 0}(2 P)$, i.e., $\Delta m \approx 90 \mathrm{MeV}$. According to the measured mass of $\chi_{c 2}(2 P)$, one can predict that the mass of $\chi_{c 0}(2 P)$ is about $3837 \mathrm{MeV}$. Thus, assigning the $X(3915)$ as the $\chi_{c 0}(2 P)$ state is still problematic, which was also pointed out in Refs. [53-55].

To better understand the properties of the wavefunctions of the charmonium states which are important to the decays, we plot the radial probability density as a function of the interquark distance $r$ in Fig. 1] It is found that the spindependent potentials have notable corrections to the $S$ - and triplet $P$-wave states. The spin-spin potential $H_{S S}$ brings an obvious splitting to the wavefunctions between $n^{1} S_{0}$ and $n^{3} S_{1}$, while the tensor potential $H_{T}$ brings notable splittings to the wavefunctions between the triplet $P$-wave states. The spin-dependent potentials only give a tiny correction to wavefunctions of the higher triplet $n D, n F, \ldots$ states. On the other hand, comparing the results from the linear potential model with those from the screened potential model, we find that for the low-lying $1 S, 2 S 1 P$ and $1 D$ charmonium states the wavefuctions obtained from both of the models are less different. However, for the higher charmonium states $n S(n \geq 3)$, $n P, n D \ldots(n \geq 2)$, the wavefuctions obtained from these two models show a notable difference.

\section{ELECTROMAGNETIC TRANSITIONS WITH HIGHER MULTIPOLE CONTRIBUTIONS}

Using the wavefunctions obtained from both the linear and screened potential models, we further study the EM transitions between charmonium states with higher multipole contributions. The EM decay properties not only are crucial for
TABLE III: Hyperfine and fine splittings in units of MeV for charmonia. LP and SP stand our results obtained from the linear potential and screened potential models, respectively. The experimental data are taken from the PDG [6]. The theoretical predictions with the previous screened potential model [11], GI model and nonrelativistic linear potential model [8], are also listed for comparison.

\begin{tabular}{c|cccccc}
\hline \hline Splitting & LP & SP & SNR [11] & NR [8] & GI [8] & Exp. [6] \\
\hline$m\left(1^{3} S_{1}\right)-m\left(1^{1} S_{0}\right)$ & 114 & 113 & 118 & 108 & 113 & $113.3 \pm 0.7$ \\
$m\left(2^{3} S_{1}\right)-m\left(2^{1} S_{0}\right)$ & 44 & 43 & 50 & 42 & 53 & $46.7 \pm 1.3$ \\
$m\left(3^{3} S_{1}\right)-m\left(3^{1} S_{0}\right)$ & 30 & 26 & 31 & 29 & 36 & \\
$m\left(4^{3} S_{1}\right)-m\left(4^{1} S_{0}\right)$ & 22 & 17 & & 22 & 25 & \\
$m\left(5^{3} S_{1}\right)-m\left(5^{1} S_{0}\right)$ & 21 & 13 & & & & \\
$m\left(1^{3} P_{2}\right)-m\left(1^{3} P_{1}\right)$ & 36 & 32 & 44 & 51 & 40 & $45.5 \pm 0.2$ \\
$m\left(1^{3} P_{1}\right)-m\left(1^{3} P_{0}\right)$ & 101 & 106 & 77 & 81 & 65 & $95.9 \pm 0.4$ \\
$m\left(2^{3} P_{2}\right)-m\left(2^{3} P_{1}\right)$ & 30 & 23 & 36 & 47 & 26 & \\
$m\left(2^{3} P_{1}\right)-m\left(2^{3} P_{0}\right)$ & 68 & 66 & 59 & 53 & 37 & \\
$m\left(3^{3} P_{2}\right)-m\left(3^{3} P_{1}\right)$ & 25 & 19 & 30 & 46 & 20 & \\
$m\left(3^{3} P_{1}\right)-m\left(3^{3} P_{0}\right)$ & 51 & 46 & 47 & 69 & 25 & \\
\hline \hline
\end{tabular}

us to determine the quantum numbers of the newly observed charmonium states, but also provide very useful references for our search for the missing charmonium states in experiments.

\section{A. The model}

The quark-photon EM coupling at the tree level is described by

$$
H_{e}=-\sum_{j} e_{j} \bar{\psi}_{j} \gamma_{\mu}^{j} A^{\mu}(\mathbf{k}, \mathbf{r}) \psi_{j},
$$

where $\psi_{j}$ stands for the $j$-th quark field in a hadron. The photon has three momentum $\mathbf{k}$, and the constituent quark $\psi_{j}$ carries a charge $e_{j}$.

In this work, the wave functions are calculated nonrelativistically from the potential models. To match the nonrelativistic wave functions of hadrons, we should adopt the nonrelativistic form of Eq. (8) in the calculations. Including the effects of binding potential between quarks [56], the nonrelativistic expansion of $H_{e}$ may be written as [57-59]

$$
h_{e} \simeq \sum_{j}\left[e_{j} \mathbf{r}_{j} \cdot \boldsymbol{\epsilon}-\frac{e_{j}}{2 m_{j}} \sigma_{j} \cdot(\boldsymbol{\epsilon} \times \hat{\mathbf{k}})\right] e^{-i \mathbf{k} \cdot \mathbf{r}_{j}},
$$

where $m_{j}, \boldsymbol{\sigma}_{j}$, and $\mathbf{r}_{j}$ stand for the constituent mass, Pauli spin vector, and coordinate for the $j$-th quark, respectively. The vector $\boldsymbol{\epsilon}$ is the polarization vector of the photon. It is found that the first and second terms in Eq. (9) are responsible for the electric and magnetic transitions, respectively. The second term in Eq. (9) is the same as that used in Ref. [7], while the first term in Eq. (9] differs from $\left(1 / m_{j}\right) \mathbf{p}_{j} \cdot \boldsymbol{\epsilon}$ used in Ref. [7] for the effects of the binding potential is included in the transition operator. This nonrelativistic EM transition operator has been widely applied to meson photoproduction reactions [59-71]. 
Finally the standard helicity transition amplitude $\mathcal{A}_{\lambda}$ between the initial state $|J \lambda\rangle$ and final state $\left|J^{\prime} \lambda^{\prime}\right\rangle$ can be calculated by

$$
\mathcal{A}_{\lambda}=-i \sqrt{\frac{\omega_{\gamma}}{2}}\left\langle J^{\prime} \lambda^{\prime}\left|h_{e}\right| J \lambda\right\rangle
$$

where $\omega_{\gamma}$ is the photon energy. It is easily found that the helicity amplitudes for the electric and magnetic operators are

$$
\begin{aligned}
& \mathcal{A}_{\lambda}^{E}=-i \sqrt{\frac{\omega_{\gamma}}{2}}\left\langle J^{\prime} \lambda^{\prime}\left|\sum_{j} e_{j} \mathbf{r}_{j} \cdot \boldsymbol{\epsilon} e^{-i \mathbf{k} \cdot \mathbf{r}_{j}}\right| J \lambda\right\rangle \\
& \mathcal{A}_{\lambda}^{M}=+i \sqrt{\frac{\omega_{\gamma}}{2}}\left\langle J^{\prime} \lambda^{\prime}\left|\sum_{j} \frac{e_{j}}{2 m_{j}} \sigma_{j} \cdot(\boldsymbol{\epsilon} \times \hat{\mathbf{k}}) e^{-i \mathbf{k} \cdot \mathbf{r}_{j}}\right| J \lambda\right\rangle \cdot(
\end{aligned}
$$

In the initial-hadron-rest system for the radiative decay precess, the momentum of the initial hadron is $\mathbf{P}_{\mathbf{i}}=\mathbf{0}$, and that of the final hadron state is $\mathbf{P}_{\mathbf{f}}=-\mathbf{k}$. Without losing generals, we select the photon momentum along the $z$ axial $(\mathbf{k}=k \hat{\mathbf{z}})$, and take the polarization vector of the photon with the right-hand form, i.e., $\boldsymbol{\epsilon}=-\frac{1}{\sqrt{2}}(1, i, 0)$, in our calculations. To easily work out the EM transition matrix elements, we use the multipole expansion of the plane wave

$$
e^{-i \mathbf{k} \cdot \mathbf{r}_{j}}=e^{-i k z_{j}}=\sum_{l} \sqrt{4 \pi(2 l+1)}(-i)^{l} j_{l}\left(k r_{j}\right) Y_{l 0}(\Omega),
$$

where $j_{l}(x)$ is the Bessel function, and $Y_{l m}(\Omega)$ are the wellknown spherical harmonics. Then, we obtain the matrix element for the electric multipole transitions with angular momentum $l$ (El transitions) [72]:

$$
\begin{aligned}
\mathcal{A}_{\lambda}^{\mathrm{E} l} & =\sqrt{\frac{\omega_{\gamma}}{2}}\left\langle J^{\prime} \lambda^{\prime}\left|\sum_{j}(-i)^{l} B_{l} e_{j} j_{l+1}\left(k r_{j}\right) r_{j} Y_{l 1}\right| J \lambda\right\rangle \\
& +\sqrt{\frac{\omega_{\gamma}}{2}}\left\langle J^{\prime} \lambda^{\prime}\left|\sum_{j}(-i)^{l} B_{l} e_{j} j_{l-1}\left(k r_{j}\right) r_{j} Y_{l 1}\right| J \lambda\right\rangle,
\end{aligned}
$$

where $B_{l} \equiv \sqrt{\frac{2 \pi l(l+1)}{2 l+1}}$. We also obtain the matrix element from the magnetic part with angular momentum $l$ ( $\mathrm{M} l$ transitions):

$$
\begin{aligned}
& \mathcal{A}_{\lambda}^{\mathrm{M} l}=\sqrt{\frac{\omega_{\gamma}}{2}}\left\langle J^{\prime} \lambda^{\prime}\left|\sum_{j}(-i)^{l} C_{l} \frac{e_{j}}{2 m_{j}} j_{l-1}\left(k r_{j}\right) \sigma_{j}^{+} Y_{l-1} 0\right| J \lambda\right\rangle \\
= & \sqrt{\frac{\omega_{\gamma}}{2}}\left\langle J^{\prime} \lambda^{\prime}\left|\sum_{j}(-i)^{l} C_{l} \frac{e_{j}}{2 m_{j}} j_{l-1}\left(k r_{j}\right)\left[\sigma_{j}^{+} \otimes Y_{l-1} 0\right]_{1}^{l}\right| J \lambda\right\rangle \\
+ & \sqrt{\frac{\omega_{\gamma}}{2}}\left\langle J^{\prime} \lambda^{\prime}\left|\sum_{j}(-i)^{l} C_{l} \frac{e_{j}}{2 m_{j}} j_{l-1}\left(k r_{j}\right)\left[\sigma_{j}^{+} \otimes Y_{l-1}\right]_{1}^{l-1}\right| J \lambda\right\rangle(15)
\end{aligned}
$$

where $C_{l} \equiv i \sqrt{8 \pi(2 l-1)}$, and $\sigma^{+}=\frac{1}{2}\left(\sigma_{x}+i \sigma_{y}\right)$ is the spin shift operator. Obviously, the $\mathrm{E} l$ transitions satisfy the parity selection rule: $\pi_{i} \pi_{f}=(-1)^{l}$; while the $\mathrm{M} l$ transitions satisfy the parity selection rule: $\pi_{i} \pi_{f}=(-1)^{l+1}$, where $\pi_{i}$ and $\pi_{f}$ stand for the parities of the initial and final hadron states, respectively. Finally, using the parity selection rules, one can express the EM helicity amplitude $\mathcal{A}$ with the matrix elements of EM multipole transitions in a unified form:

$$
\mathcal{A}_{\lambda}=\sum_{l}\left\{\frac{1+(-1)^{\pi_{i} \pi_{f}+l}}{2} \mathcal{A}_{\lambda}^{\mathrm{E} l}+\frac{1-(-1)^{\pi_{i} \pi_{f}+l}}{2} \mathcal{A}_{\lambda}^{\mathrm{M} l}\right\} .
$$

Combining the parity selection rules, we easily know the possible EM multipole contributions to a EM transition considered in present work, which are listed in Tab. IV.

It should be pointed out that, the second term of Eq. 15) from the magnetic part is included into the electric part by the most general decomposition of the helicity amplitudes [7375]:

$$
\mathcal{A}_{\lambda}=\sum_{k \geq 1}(-1)^{k+1} \sqrt{\frac{2 k+1}{2 J+1}} a_{k}\left\langle k-1 ; J^{\prime} \lambda+1 \mid J \lambda\right\rangle,
$$

with $a_{k}$ corresponding to the multipole amplitude of the EM tensor operators with a rank $k$. The second term of Eq. 15) is called as "extra" electric-multipole term, $E_{R}$, by F. E. Close $e t$ al. [76]. Specifically, for ${ }^{3} S_{1} \leftrightarrow \leftrightarrow^{3} P_{1}$ :

$$
\begin{aligned}
& a_{1}=E_{1}+E_{R}=-\frac{\sqrt{2}}{2}\left(\mathcal{A}_{0}+\mathcal{A}_{-1}\right), \\
& a_{2}=M_{2}=-\frac{\sqrt{2}}{2}\left(\mathcal{A}_{0}-\mathcal{A}_{-1}\right) ;
\end{aligned}
$$

for ${ }^{3} P_{2} \rightarrow{ }^{3} S_{1}$ :

$$
\begin{array}{r}
a_{1}=E_{1}+E_{R}=\frac{\sqrt{10}}{2}\left(\sqrt{3} \mathcal{A}_{-1}-\mathcal{A}_{0}\right), \\
a_{2}=M_{2}=\frac{\sqrt{6}}{2}\left(\sqrt{3} \mathcal{A}_{0}-\mathcal{A}_{-1}\right) ;
\end{array}
$$

and for ${ }^{3} S_{1} \rightarrow{ }^{3} P_{2}$ :

$$
\begin{array}{r}
a_{1}=E_{1}+E_{R}=\frac{\sqrt{10}}{2}\left(\sqrt{3} \mathcal{A}_{0}-\mathcal{A}_{-1}\right), \\
a_{2}=M_{2}=\frac{\sqrt{6}}{2}\left(\mathcal{A}_{0}-\sqrt{3} \mathcal{A}_{-1}\right) .
\end{array}
$$

Here $E_{1}$ is the leading electric-dipole term determined by Eq.(14), and $M_{2}$ is the magnetic-quadrupole term related to the first term of Eq. (15). It should be mentioned that we have $a_{3}=0$ for the above transitions in present work.

TABLE IV: Possible EM multipole contributions to a EM transition between two charmonium states.

\begin{tabular}{lc}
\hline \hline \multicolumn{1}{c}{ process } & multipole contribution \\
\hline$n^{3} S_{1} \longleftrightarrow m^{1} S_{0}$ & $\mathrm{M} 1$ \\
$n^{3} P_{J} \longleftrightarrow m^{3} S_{1}$ & E1, M2 \\
$n^{1} P_{1} \longleftrightarrow m^{1} S_{0}$ & E1 \\
$n^{3} D_{J} \longleftrightarrow m^{3} P_{J}$ & E1, E3, M2, M4 \\
$n^{1} D_{1} \longleftrightarrow m^{1} P_{1}$ & E1, E3 \\
$n^{3} P_{J} \longleftrightarrow m^{1} P_{1}$ & $\mathrm{M} 1, \mathrm{M} 3$ \\
\hline \hline
\end{tabular}


Then, the partial decay widths of the EM transitions are given by

$$
\begin{aligned}
\Gamma & =\frac{|\mathbf{k}|^{2}}{\pi} \frac{2}{2 J_{i}+1} \frac{M_{f}}{M_{i}} \sum_{\lambda}\left|\mathcal{A}_{\lambda}\right|^{2} \\
& =\frac{|\mathbf{k}|^{2}}{\pi} \frac{2}{2 J_{i}+1} \frac{M_{f}}{M_{i}} \sum_{k}\left|a_{k}\right|^{2},
\end{aligned}
$$

where $J_{i}$ is the total angular momenta of the initial mesons, $J_{f z}$ and $J_{i z}$ are the components of the total angular momentum along the $z$ axis of initial and final mesons, respectively. To take into account of the relativistic effects, following the idea of Ref. [8], we introduce an overall relativistic phase space factor $E_{f} / M_{i}$ in our predictions of the widths, which is usually not far from unity. $M_{f}$ and $E_{f}$ stand for the mass and total energy of the final charmonium state, respectively. $M_{i}$ is the mass of the initial charmonium state.

Finally, we should mention that in the most general decomposition of the helicity amplitudes [73-75], the $a_{k}$ is considered as the magnetic or electric multipole amplitude, thus, in the total decay width the electric and magnetic multipole amplitudes can not interfere with each other. However, the "extra" electric-multipole term comes from the magnetic part, thus, in this sense the electric-magnetic interference term appears in the total decay width [76-78].

\section{B. Results and discussions}

\section{Lighter states}

First, we calculate the M1 transitions of the low-lying $1 S$, $2 S$ and $3 S$ states. Our results compared with experimental data and other model predictions have been listed in Tab. V Both our linear and screened potential model calculations obtain a compatible prediction. Our predictions are consistent with those of NR and GI models [8]. It should be pointed out that our predictions together with those in the framework of GI and NR potential models [8] give a very large partial width for the $\psi(2 S) \rightarrow \eta_{c}(1 S) \gamma$ process, which is about an order of magnitude larger than the world average data from the PDG [6] and the prediction of $\Gamma\left[\psi(2 S) \rightarrow \eta_{c}(1 S) \gamma\right] \simeq 0.4(8)$ $\mathrm{keV}$ from Lattice QCD [20]. Although our prediction of $\Gamma\left[J / \psi \rightarrow \eta_{c}(1 S) \gamma\right]$ is obviously larger than the PDG average data [6], it is in agreement with the recent measurement $\Gamma\left[J / \psi \rightarrow \eta_{c}(1 S) \gamma\right] \simeq 2.98 \pm 0.18_{-0.33}^{+0.15} \mathrm{keV}$ at KEDR [79]. As a whole, strong model dependence exists in the predictions of the M1 transitions, more studies are needed in both theory and experiments.

Then, we calculate the E1 dominant radiative decays of the $1 P$ and $2 S$ states. Our results compared with experimental data and other model predictions have been listed in Tab. VI. Both our linear and screened potential model calculations obtain a compatible prediction, because the wave-functions and masses for the low-lying states from these two models have less differences. Our predictions are reasonable agreement with the data. The predictions from different models are con- sistent with each other in a magnitude, although there are differences more or less.

\section{2. $\psi(3770), X(3823)$ and the missing $1 D$ states}

The $\psi(3770)$ resonance is primarily a $\psi_{1}(1 D)$ state with small admixtures of $\psi(2 S)$ [1]. It can decay into $\chi_{c J}(1 P) \gamma$. These decay processes are dominated by the E1 transition. The radiative decays of $\psi(3770)$ are still not well understood. For example, the predictions of $\Gamma\left[\psi(3770) \rightarrow \chi_{c 0}(1 P) \gamma\right]$ vary in a very large range $(200,500) \mathrm{keV}$ [45]. Considering $\psi(3770)$ as a pure $\psi_{1}(1 D)$ state, we calculate the radiative decay widths of $\Gamma\left[\psi(3770) \rightarrow \chi_{c J}(1 P) \gamma\right]$ with the wavefunctions obtained from the linear and screened potential models, respectively. Our results have been listed in Tab. IX From the table, we can see that both models give very similar predictions for the partial decay widths. Considering the leading E1 decays only, our predictions are in agreement with the world average data within their uncertainties [6]. However, including the magnetic part, the partial decay widths predicted by us are about a factor of 1.5 larger than the world average data [6] and the recent measurements from BESIII [45, 80]. It is unclear whether these discrepancies are caused by our model limitations or come from the experimental uncertainties. It should be mentioned that although some predictions from the models with a relativistic assumption [8, 11] or a coupled-channel correction [30] seem to better agree quantitatively with the experimental data, however, the corrections of the magnetic part are not included in their calculations. To better understand the radiative decay properties of $\psi(3770)$, more studies are needed in both theory and experiments.

Recently, $X(3823)$ as a good candidate of $\psi_{2}(1 D)$ was observed by the Belle Collaboration in the $B \rightarrow \chi_{c 1} \gamma K$ decay with a statistical significance of $3.8 \sigma[81]$. Lately, this state was confirmed by the BESIII Collaboration in the process $e^{+} e^{-} \rightarrow \pi^{+} \pi^{-} X(3823) \rightarrow \pi^{+} \pi^{-} \chi_{c 1} \gamma$ with a statistical significance of $6.2 \sigma$ [82]. Assigning $X(3823)$ as the $\psi_{2}(1 D)$ state, we predict the radiative decay widths of $\Gamma[X(3823) \rightarrow$ $\left.\chi_{c J}(1 P) \gamma\right]$. Both the linear and screened potential models give quite similar predictions,

$$
\begin{aligned}
& \Gamma\left[X(3823) \rightarrow \chi_{c 1}(1 P) \gamma\right] \simeq 300 \mathrm{keV}, \\
& \Gamma\left[X(3823) \rightarrow \chi_{c 2}(1 P) \gamma\right] \simeq 90 \mathrm{keV} .
\end{aligned}
$$

Our prediction of $\Gamma\left[X(3823) \rightarrow \chi_{c 1}(1 P) \gamma\right]$ is close to the predictions in Refs. [8, 11, 37, 83], while our prediction for $\Gamma\left[X(3823) \rightarrow \chi_{c 2}(1 P) \gamma\right]$ is about a factor of 1.4 1.8 larger than the predictions in these works. Furthermore, our predicted partial width ratio,

$$
\frac{\Gamma\left[X(3823) \rightarrow \chi_{c 2}(1 P) \gamma\right]}{\Gamma\left[X(3823) \rightarrow \chi_{c 1}(1 P) \gamma\right]} \simeq 30 \%
$$

is consistent with the observations $<42 \%$ [82]. The $X(3823)$ state mainly decays into the $\chi_{c 1,2}(1 P) \gamma, J / \psi \pi \pi$ and $g g g$ channels. The predicted partial widths for $J / \psi \pi \pi$ and $g g g$ channels are about $(210 \pm 110) \mathrm{keV}$ and $80 \mathrm{keV}$, respectively [14]. Thus, 
the total width of the $X(3823)$ might be $\Gamma_{\text {tot }} \simeq 680 \pm 110 \mathrm{keV}$, from which we obtain large branching ratios

$$
\begin{aligned}
& B r\left[X(3823) \rightarrow \chi_{c 1}(1 P) \gamma\right] \simeq 42 \% \\
& \operatorname{Br}\left[X(3823) \rightarrow \chi_{c 2}(1 P) \gamma\right] \simeq 13 \%
\end{aligned}
$$

The large branching fraction $\operatorname{Br}\left[X(3823) \rightarrow \chi_{c 1}(1 P) \gamma\right]$ can explain why the $X(3823)$ was first observed in the $\chi_{c 1} \gamma$ channel.

Another two $1 D$-wave states $\psi_{3}(1 D)$ and $\eta_{c 2}(1 D)$ have not been observed in experiments. According to the theoretical predictions, their masses are very similar to that of $\psi_{2}(1 D)$. If $X(3823)$ corresponds to the $\psi_{2}(1 D)$ state indeed, the masses of the $\psi_{3}(1 D)$ and $\eta_{c 2}(1 D)$ resonances should be around 3.82 $\mathrm{GeV}$. For the singlet $1 D$ state $\eta_{c 2}(1 D)$, its main radiative transition is $\eta_{c 2}(1 D) \rightarrow h_{c}(1 P) \gamma$. This process is governed by the E1 transition, the effects from the E3 transition are negligibly small. Taking the mass of $\eta_{c 2}(1 D)$ with $M=3820 \mathrm{MeV}$, with the wavefunctions calculated from the linear potential model we predict that

$$
\Gamma\left[\eta_{c 2}(1 D) \rightarrow h_{c}(1 P) \gamma\right] \simeq 362 \mathrm{keV}
$$

which is consistent with that of the screened potential model. Our results are close to the previous predictions in Refs. [8, 11] (see Tab. IX . Combined the predicted partial widths of the other two main decay modes $g g$ and $\eta_{c} \pi \pi$ [14], the total width of $\eta_{c 2}(1 D)$ is estimated to be $\Gamma_{\text {tot }} \simeq 760 \mathrm{keV}$. Then, we can obtain a large branching ratio

$$
\operatorname{Br}\left[\eta_{c 2}(1 D) \rightarrow h_{c}(1 P) \gamma\right] \simeq 48 \% \text {. }
$$

Combining the measured branching ratios of $\operatorname{Br}\left[h_{c}(1 P) \rightarrow\right.$ $\left.\eta_{c} \gamma\right] \simeq 51 \%$ and $B r\left[\eta_{c} \rightarrow K \bar{K} \pi\right] \simeq 7.3 \%$ [6], we obtain

$$
\operatorname{Br}\left[\eta_{c 2}(1 D) \rightarrow h_{c}(1 P) \gamma \rightarrow \eta_{c} \gamma \gamma \rightarrow K \bar{K} \pi \gamma \gamma\right] \simeq 1.8 \%
$$

It should be mentioned that the $\eta_{c 2}(1 D)$ state could be produced via the $B \rightarrow \eta_{c 2}(1 D) K$ process as suggested in Refs. [13, 84, 85]. The expectations are to accumulate $10^{10}$ $\Upsilon(4 S) B \bar{B}$ events at Belle [2, 9]. If the branching fraction $\mathrm{Br}\left[B \rightarrow \eta_{c 2}(1 D) K\right]$ is $O\left(10^{-5}\right)$ as predicted in [84], the $10^{10}$ $B \bar{B}$ events could let us observe $O(1000) \eta_{c 2}(1 D)$ events via the two-photon cascade $\eta_{c 2}(1 D) \rightarrow h_{c}(1 P) \gamma \rightarrow \eta_{c} \gamma \gamma$ in the $\gamma \gamma K \bar{K} \pi$ final states.

While for the triplet $1 D$ state $\psi_{3}(1 D)$, its common radiative transition is $\psi_{3}(1 D) \rightarrow \chi_{c 2}(1 P) \gamma$. Taking the mass of $\psi_{3}(1 D)$ with $M=3830 \mathrm{MeV}$, we calculate the partial decay widths $\Gamma\left[\psi_{3}(1 D) \rightarrow \chi_{c 2}(1 P) \gamma\right]$ with both the linear and screened potential models. Both of the models give a very similar result

$$
\Gamma\left[\psi_{3}(1 D) \rightarrow \chi_{c 2}(1 P) \gamma\right] \simeq 350 \mathrm{keV}
$$

The magnitude of the partial decay width of $\Gamma\left[\psi_{3}(1 D) \rightarrow\right.$ $\left.\chi_{c 2}(1 P) \gamma\right]$ predicted by us is compatible with that in Refs. [8, 11, 37]. Combining the predicted total width $\Gamma_{\text {tot }} \simeq 3 \mathrm{MeV}$ for $\psi_{3}(1 D)[14$, we estimate the branching ratio

$$
\operatorname{Br}\left[\psi_{3}(1 D) \rightarrow \chi_{c 2}(1 P) \gamma\right] \simeq 12 \%
$$

The missing $\psi_{3}(1 D)$ might be produced via the $B \rightarrow \psi_{3}(1 D) K$ process at Belle [13, 84, 86], and reconstructed in the $\chi_{c 2}(1 P) \gamma$ decay mode with $\chi_{c 2}(1 P) \rightarrow J / \psi \gamma$ and $J / \psi \rightarrow \mu^{+} \mu^{-} / e^{+} e^{-}$. If the branching fraction $\operatorname{Br}\left[B \rightarrow \psi_{3}(1 D) K\right]$ is $O\left(10^{-5}\right)[84$, 86], based on the $10^{10} B \bar{B}$ data to be accumulated at Belle II, we expect that $O(100) \psi_{3}(1 D)$ events could reconstructed in the $\chi_{c 2}(1 P) \gamma$ channel with $\chi_{c 2}(1 P) \rightarrow J / \psi \gamma$ and $J / \psi \rightarrow$ $\mu^{+} \mu^{-} / e^{+} e^{-}$.

\section{3. $X(3872,3915)$ and the $2 P$ states}

In the $2 P$-wave states, only $\chi_{c 2}(2 P)$ has been established experimentally. This state was observed by both Belle [87] and BaBar [88] in the two-photon fusion process $\gamma \gamma \rightarrow D \bar{D}$ with a mass $M \simeq 3927 \mathrm{MeV}$ and a narrow width $\Gamma \simeq 24$ $\mathrm{MeV}$ [6]. We analyze its radiative transitions to $\psi(1 D) \gamma, J / \psi \gamma$ and $\psi(2 S) \gamma$. Both the linear and screened potential give very similar predictions:

$$
\begin{aligned}
\Gamma\left[\chi_{c 2}(2 P) \rightarrow \psi(3770) \gamma\right] & \simeq 0.4 \mathrm{keV}, \\
\Gamma\left[\chi_{c 2}(2 P) \rightarrow \psi_{2}(1 D) \gamma\right] & \simeq 3.2 \mathrm{keV}, \\
\Gamma\left[\chi_{c 2}(2 P) \rightarrow \psi_{3}(1 D) \gamma\right] & \simeq 20 \mathrm{keV} .
\end{aligned}
$$

Our predictions are notablely different from those of the NR potential model [8] (see Tab.VIII). With the measured width, we further predicted the branching ratios

$$
\begin{aligned}
\operatorname{Br}\left[\chi_{c 2}(2 P) \rightarrow \psi(3770) \gamma\right] & \simeq 1.7 \times 10^{-5}, \\
\operatorname{Br}\left[\chi_{c 2}(2 P) \rightarrow \psi_{2}(1 D) \gamma\right] & \simeq 1.3 \times 10^{-4}, \\
\operatorname{Br}\left[\chi_{c 2}(2 P) \rightarrow \psi_{3}(1 D) \gamma\right] & \simeq 1.5 \times 10^{-3} .
\end{aligned}
$$

Combining these ratios with the decay properties of $\psi(1 D)$ and $\chi_{c}(1 P)$ states, we estimate the combined branching ratios for the decay chains $\chi_{c 2}(2 P) \rightarrow \psi(1 D) \gamma \rightarrow \chi_{c}(1 P) \gamma \gamma \rightarrow$ $J / \psi \gamma \gamma \gamma$, our results have been listed in Tab. X It is found that the most important decay chains involving $\psi_{2}(1 D)$ and $\psi_{3}(1 D)$ are $\chi_{c 2}(2 P) \rightarrow \psi_{2}(1 D) \gamma \rightarrow \chi_{c 1}(1 P) \gamma \gamma \rightarrow J / \psi \gamma \gamma \gamma$ $\left(B r \simeq 1.9 \times 10^{-5}\right)$ and $\chi_{c 2}(2 P) \rightarrow \psi_{3}(1 D) \gamma \rightarrow \chi_{c 2}(1 P) \gamma \gamma \rightarrow$ $J / \psi \gamma \gamma \gamma\left(B r \simeq 1.4 \times 10^{-5}\right)$. These decay chains might be hard observed at present because the very small production cross section of $\chi_{c 2}(2 P)$.

The $\chi_{c 2}(2 P)$ state has relatively larger radiative decay rates into $J / \psi \gamma$ and $\psi(2 S) \gamma$. With the linear potential model, we obtain

$$
\begin{aligned}
\Gamma\left[\chi_{c 2}(2 P) \rightarrow J / \psi \gamma\right] & \simeq 93 \mathrm{keV}, \\
\Gamma\left[\chi_{c 2}(2 P) \rightarrow \psi(2 S) \gamma\right] & \simeq 135 \mathrm{keV},
\end{aligned}
$$

which are consistent with those of the screened potential model. Combined the measured width, the branching ratios are predicted to be

$$
\begin{aligned}
\operatorname{Br}\left[\chi_{c 2}(2 P) \rightarrow J / \psi \gamma\right] & \simeq 3.9 \times 10^{-3}, \\
\operatorname{Br}\left[\chi_{c 2}(2 P) \rightarrow \psi(2 S) \gamma\right] & \simeq 5.6 \times 10^{-3} .
\end{aligned}
$$

It is might be a challenge to observe $\chi_{c 2}(2 P)$ in the $J / \psi \gamma$ and $\psi(2 S) \gamma$ channels with $J / \psi / \psi(2 S) \rightarrow \mu^{+} \mu^{-}$at BESIII. For example, we produce $\chi_{c 2}(2 P)$ via the process $e^{+} e^{-} \rightarrow \gamma \chi_{c 2}(2 P)$ 
at BESIII. Based on the cross section $\sim 0.2 \mathrm{pb}$ predicted in Ref. [89], we estimate that even if BESIII can collect $10 \mathrm{fb}^{-1}$ data sample above open charm threshold, we only accumulate about $2000 e^{+} e^{-} \rightarrow \gamma \chi_{c 2}(2 P)$ events. Combining our predictions of branching ratios $\operatorname{Br}\left[\chi_{c 2}(2 P) \rightarrow J / \psi \gamma \rightarrow \gamma \mu^{+} \mu^{-}\right] \sim$ $O\left(10^{-4}\right)$, we find there is less hope for observing the radiative decay modes of $\chi_{c 2}(2 P)$ at BESIII. It should be mentioned that the decay chain $\chi_{c 2}(2 P) \rightarrow J / \psi \gamma, \psi(2 S) \gamma \rightarrow \gamma \mu^{+} \mu^{-}$might be observed at Belle II or LHCb via the $B \rightarrow \chi_{c 2}(2 P) X$ decay. The expectations are to accumulate $10^{10} \Upsilon(4 S) B \bar{B}$ events at Belle [2, 9]. If the branching ratio of $B r\left[B \rightarrow \chi_{c 2}(2 P) X\right] \sim$ $10^{-5}$, we may observed $O(10) \chi_{c 2}(2 P)$ 's in the decay chain $\chi_{c 2}(2 P) \rightarrow J / \psi \gamma \rightarrow \gamma \mu^{+} \mu^{-}$.

The $\chi_{c 1}(2 P)$ state is still not established in experiments. According to the fine splitting between $\chi_{c 2}(2 P)$ and $\chi_{c 1}(2 P)$, we estimate the mass of $\chi_{c 1}(2 P)$ is around $M=3900 \mathrm{MeV}$. With this mass we calculate the transitions of $\chi_{c 1}(2 P)$ into $\psi(2 S) \gamma, J / \psi \gamma, \psi_{1}(1 D) \gamma$, and $\psi_{2}(1 D) \gamma$. Our results are listed in Tab. VIII With the wavefuctions obtained from the linear potential model, it is found that the partial widths

$$
\begin{aligned}
\Gamma\left[\chi_{c 1}(2 P) \rightarrow J / \psi \gamma\right] & \simeq 81 \mathrm{keV}, \\
\Gamma\left[\chi_{c 1}(2 P) \rightarrow \psi(2 S) \gamma\right] & \simeq 139 \mathrm{keV},
\end{aligned}
$$

are slightly smaller than those from the screened potential model. Combined the predicted width in Ref. [8], the branching ratios might be

$$
\begin{aligned}
\operatorname{Br}\left[\chi_{c 1}(2 P) \rightarrow J / \psi \gamma\right] & \simeq 4.9 \times 10^{-4}, \\
\operatorname{Br}\left[\chi_{c 1}(2 P) \rightarrow \psi(2 S) \gamma\right] & \simeq 8.4 \times 10^{-4} .
\end{aligned}
$$

The partial widths of $\Gamma\left[\chi_{c 1}(2 P) \rightarrow \psi_{1,2}(1 D) \gamma\right]$ are about several $\mathrm{keV}$, their branching ratios are $O\left(10^{-5}\right)$.

The $X(3872)$ resonance has the same quantum numbers as $\chi_{c 1}(2 P)$ (i.e., $J^{P C}=1^{++}$) and a similar mass to the predicted value of $\chi_{c 1}(2 P)$. However, its exotic properties can not be well understood with a pure $\chi_{c 1}(2 P)$ state [4, 90]. To understand the nature of $X(3872)$, measurements of the radiative decays of $X(3872)$ have been carried out by the BaBar [91], Belle [92], and LHCb [93] collaborations, respectively. Obvious evidence of $X(3872) \rightarrow J / \psi \gamma$ was observed by these collaborations. Furthermore, the BaBar and LHCb Collaborations also observed evidence of $X(3872) \rightarrow \psi(2 S) \gamma$. The branching fraction ratio

$$
R_{\psi^{\prime} \gamma / \psi \gamma}^{\exp }=\frac{\Gamma[X(3872) \rightarrow \psi(2 S) \gamma]}{\Gamma[X(3872) \rightarrow J / \psi \gamma]} \simeq 3.4 \pm 1.4,
$$

obtained by the BaBar Collaboration [91] is consistent with the recent measurement $R_{\psi^{\prime} \gamma / \psi \gamma}^{\exp }=2.46 \pm 0.93$ of LHCb Collaboration [93].

Considering $X(3872)$ as a pure $\chi_{c 1}(2 P)$ state, we calculate the radiative decays $X(3872) \rightarrow J / \psi \gamma, \psi(2 S) \gamma$. With the linear potential model, we predict that

$$
\begin{aligned}
\Gamma[X(3872) \rightarrow J / \psi \gamma) & \simeq 72 \mathrm{keV}, \\
\Gamma[X(3872) \rightarrow \psi(2 S) \gamma] & \simeq 94 \mathrm{keV} .
\end{aligned}
$$

With these predicted partial widths, we can easily obtain the ratio

$$
R_{\psi^{\prime} \gamma / \psi \gamma}^{\mathrm{th}}=\frac{\Gamma[X(3872) \rightarrow \psi(2 S) \gamma]}{\Gamma[X(3872) \rightarrow J / \psi \gamma]} \simeq 1.3
$$

which is slightly smaller than the lower limit of the measurements from the BaBar [91] and LHCb [93]. Our predictions from the screened potential model are consistent with those from the linear potential model. Thus, from the view of branching fraction ratio $R_{\psi^{\prime} \gamma / \psi \gamma}$, we can not exclude the $X(3872)$ as a candidate of $\chi_{c 1}(2 P)$.

On the other hand, if $X(3872)$ corresponds to $\chi_{c 1}(2 P)$, with the measured width (about several MeV) [6], we can estimate

$$
\begin{aligned}
\operatorname{Br}[X(3872) \rightarrow J / \psi \gamma] & \sim O\left(10^{-2}\right), \\
\operatorname{Br}[X(3872) \rightarrow \psi(2 S) \gamma] & \sim O\left(10^{-2}\right) .
\end{aligned}
$$

Combining the branching ratio $B r\left[B \rightarrow \chi_{c 1}(2 P) K\right] \sim O\left(10^{-4}\right)$ predicted in Ref. [94], we can further estimate that $\operatorname{Br}[B \rightarrow$ $\left.\chi_{c 1}(2 P) K\right] \times \operatorname{Br}[X(3872) \rightarrow J / \psi \gamma / \psi(2 S) \gamma] \sim O\left(10^{-6}\right)$, which is also consistent with the Belle measurements [92].

The $\chi_{c 0}(2 P)$ state is still not well-established, although $X(3915)$ was recommended as the $\chi_{c 0}(2 P)$ state in Ref. [95], and also assigned as the $\chi_{c 0}(2 P)$ state by the PDG recently [6]. Assigning $X(3915)$ as the $\chi_{c 0}(2 P)$ state will face several serious problems [53, 54]. Recently, Zhou et al. carried out a combined amplitude analysis of the $\gamma \gamma \rightarrow D \bar{D}, \omega J / \psi$ data [55]. They demonstrated that $X(3915)$ and $X(3930)$ can be regarded as the same state with $J^{P C}=2^{++}$(i.e., $\chi_{c 2}(2 P)$ ). With the screened and linear potential models, our predicted masses for the $\chi_{c 0}(2 P)$ state are $\sim 3848 \mathrm{MeV}$ and $\sim 3869$ $\mathrm{MeV}$, respectively, which are consistent with the previous predictions in Refs. [8, 11], and the mass extracted by Guo and Meissner by refitting the BaBar and Belle data of $\gamma \gamma \rightarrow D \bar{D}$ separately [53]. The $\chi_{c 0}(2 P)$ state can decay via the radiative transitions $\chi_{c 0}(2 P) \rightarrow \psi(3770) \gamma, \psi(2 S) \gamma, J / \psi \gamma$. With the wavefunctions obtained from both the screened and linear potential models, we calculate the decay rates of these radiative transitions. Our results are listed in Tab.VIII From the table, it is found that both of the models give similar predictions. The partial width for $\chi_{c 0}(2 P) \rightarrow \psi(2 S) \gamma$ is

$$
\Gamma\left[\chi_{c 0}(2 P) \rightarrow \psi(2 S) \gamma\right] \simeq 110 \pm 10 \mathrm{keV} .
$$

The $\chi_{c 0}(2 P)$ might be very broad with a width of $\sim 200 \mathrm{MeV}$ extracted from experimental data [53], which is about an order of magnitude larger than that predicted in Ref. [8]. With the broad width, the branching ratio is predicted to be

$$
B r\left[\chi_{c 0}(2 P) \rightarrow \psi(2 S) \gamma\right] \simeq 5.5 \times 10^{-4} .
$$

It should be mentioned that there is less chance for producing the $\chi_{c 0}(2 P)$ state via the radiative decay chains $\psi(4040,4160,4415) \rightarrow \chi_{c 0}(2 P) \gamma \chi_{c 0}(2 P) \rightarrow \psi(1 S, 2 S) \gamma \gamma \rightarrow$ $\gamma \gamma \mu^{+} \mu^{-}$(see Tabs. XIXIII).

There is no information of $h_{c}(2 P)$ from experiments. According to our predictions, the mass-splitting between $\chi_{c 2}(2 P)$ and $h_{c}(2 P)$ is about $M_{\chi_{c 2}(2 P)}-M_{h_{c}(2 P)}=(26 \pm 4) \mathrm{MeV}$. Thus, the mass of $h_{c}(2 P)$ is most likely to be $M_{h_{c}(2 P)} \simeq 3900 \mathrm{MeV}$. The typical radiative decay channels of $h_{c}(2 P)$ are $\eta_{c}(1 S, 2 S) \gamma$ and $\eta_{c 2}(1 D) \gamma$. With the wavefunctions obtained from the linear and screened potentials, we further calculate these radiative decays. It is found that the radiative transition rates of $h_{c}(2 P) \rightarrow \eta_{c 2}(1 D) \gamma, \eta_{c}(1 S) \gamma$ and $\eta_{c}(2 S) \gamma$ channels are fairly 
large. Both the linear and screened potential models give very similar predictions

$$
\begin{aligned}
\Gamma\left(h_{c}(2 P) \rightarrow \eta_{c}(1 S) \gamma\right) & \simeq 135 \mathrm{keV}, \\
\Gamma\left(h_{c}(2 P) \rightarrow \eta_{c}(2 S) \gamma\right) & \simeq 160 \mathrm{keV}, \\
\Gamma\left(h_{c}(2 P) \rightarrow \eta_{c 2}(1 D) \gamma\right) & \simeq 25 \mathrm{keV} .
\end{aligned}
$$

The rather sizeable partial widths for $h_{c}(2 P) \rightarrow \eta_{c}(1 S, 2 S) \gamma$ are also obtained in the previous potential model calculations [8, 11, 18]. Combined the theoretical width $\Gamma \simeq 87 \mathrm{MeV}$ from [8], the branching ratios are predicted to be

$$
\begin{aligned}
B r\left[h_{c}(2 P) \rightarrow \eta_{c}(1 S) \gamma\right] & \simeq 1.6 \times 10^{-3}, \\
\operatorname{Br}\left[h_{c}(2 P) \rightarrow \eta_{c}(2 S) \gamma\right] & \simeq 1.8 \times 10^{-3}, \\
\operatorname{Br}\left[h_{c}(2 P) \rightarrow \eta_{c 2}(1 D) \gamma\right] & \simeq 2.8 \times 10^{-4} .
\end{aligned}
$$

The missing $h_{c}(2 P)$ state might be produced via $B \rightarrow h_{c}(2 P) K$ process, and reconstructed in the $\eta_{c}(1 S, 2 S) \gamma$ decay modes with $\eta_{c}(1 S, 2 S) \rightarrow K \bar{K} \pi$ at Belle II and LHCb.

\section{4. $\psi(4040)$ and the missing $\eta_{c}(3 S)$ state}

The $\psi(4040)$ resonance is commonly identified with the $\psi(3 S)$ state [1]. This state can decay into $\chi_{c J}(1 P) \gamma$ and $\chi_{c J}(2 P) \gamma$ via the radiative transitions. We have calculated these precesses with both the linear and screened potential model. Our results have been listed in Tab.VI In our calculations, we find that the radiative transition rates of $\psi(4040) \rightarrow$ $\chi_{c J}(1 P) \gamma$ are relatively weak. Using the PDG value for the total width $\Gamma \simeq 80 \mathrm{MeV}$ [6], we obtain the branching ratios $\operatorname{Br}\left[\psi(4040) \rightarrow \chi_{c J}(1 P) \gamma\right] \sim O\left(10^{-5}\right)$, which are consistent with the measurements $\operatorname{Br}\left[\psi(4040) \rightarrow \chi_{c 1,2}(1 P) \gamma\right]<2 \%$ [6]. Interestedly, it is found that the radiative transition rates of $\psi(4040) \rightarrow \chi_{c J}(2 P) \gamma$ are rather sizeable. The decay rates into the $\chi_{c J}(2 P) \gamma$ channels are about one order of magnitude larger than those into the $\chi_{c J}(1 P) \gamma$ channels. With the screened potential model, we obtain that

$$
\begin{aligned}
& \Gamma\left[\psi(4040) \rightarrow \chi_{c 2}(2 P) \gamma\right] \simeq 82 \mathrm{keV}, \\
& \Gamma\left[\psi(4040) \rightarrow \chi_{c 1}(2 P) \gamma\right] \simeq 67 \mathrm{keV}, \\
& \Gamma\left[\psi(4040) \rightarrow \chi_{c 0}(2 P) \gamma\right] \simeq 27 \mathrm{keV},
\end{aligned}
$$

which are about $15 \%$ larger than our linear potential model predictions. Relatively large partial decay widths for $\psi(4040) \rightarrow \chi_{C J}(2 P) \gamma$ were also found in the previous studies [8]. With the measured width $\Gamma \simeq 80 \mathrm{MeV}$ from the PDG [6], we estimate the branching ratios

$$
\begin{aligned}
& \operatorname{Br}\left[\psi(4040) \rightarrow \chi_{c 2}(2 P) \gamma\right] \simeq 1.0 \times 10^{-3}, \\
& \operatorname{Br}\left[\psi(4040) \rightarrow \chi_{c 1}(2 P) \gamma\right] \simeq 0.8 \times 10^{-3}, \\
& \operatorname{Br}\left[\psi(4040) \rightarrow \chi_{c 0}(2 P) \gamma\right] \simeq 3.3 \times 10^{-4} .
\end{aligned}
$$

It should be pointed out that BESIII plan to collect $5 \sim 10 \mathrm{fb}^{-1}$ $\psi(4040)$ in the coming years [99]. Using the cross section of $10 \mathrm{nb}$ based on BES and CLEO measurements [100102], we expect to accumulate $(0.5 \sim 1.0) \times 10^{8} \psi(4040)$ events. The $\psi(4040)$ might provide us a source to produce $\chi_{c J}(2 P)$ states via the radiative transitions. Thus, we further estimate the number of events of the two-photon cascades involving the $\chi_{c J}(2 P)$ states. The results are listed in Tab. XI From the table, we can see that about $O(10) \chi_{c 2}(2 P)$ events should be observed at BESIII via the radiative transition chain $\psi(4040) \rightarrow \chi_{c 2}(2 P) \gamma \rightarrow J / \psi \gamma \gamma \rightarrow \gamma \gamma \mu^{+} \mu^{-}$.

The $\eta_{c}(3 S)$ state is not established in experiments. According to the model predictions, the hyperfine splitting between $3^{3} S_{1}$ and $3{ }^{1} S_{0}$ is about $30 \mathrm{MeV}$ (see Tab. III). Thus, the mass of $\eta_{c}(3 S)$ is most likely to be $\sim 4010 \mathrm{MeV}$. With this mass, we calculate the radiative transitions $\eta_{c}(3 S) \rightarrow h_{c}(1 P) \gamma, h_{c}(2 P) \gamma$. Our prediction of the decay rate of $\eta_{c}(3 S) \rightarrow h_{c}(1 P) \gamma$ is tiny. However, the partial decay widths are rather sizeable, with the screened potential model we predict that

$$
\Gamma\left[\eta_{c}(3 S) \rightarrow h_{c}(2 P) \gamma\right] \simeq 130 \mathrm{keV},
$$

which is slightly $(\sim 20 \%)$ larger than our prediction with the linear potential model. Our prediction of $\Gamma\left[\eta_{c}(3 S) \rightarrow\right.$ $\left.h_{c}(2 P) \gamma\right]$ is consistent with the previous calculation in Ref. [8] (see Tab. VII . Combined the predicted width $\Gamma \simeq 80 \mathrm{MeV}$ from [8], the branching ratio of $B r\left[\eta_{c}(3 S) \rightarrow h_{c}(2 P) \gamma\right]$ is estimated to be $1.6 \times 10^{-3}$.

\section{5. $\psi(4160)$ and the missing $2 D$ states}

The $1^{--}$state $\psi(4160)$ is commonly identified with the $2^{3} D_{1}$ state. The average experimental mass and width from the PDG are $M=4191 \pm 5 \mathrm{MeV}$ and $\Gamma=70 \pm 10 \mathrm{MeV}$, respectively [6], which are consistent with linear potential model predictions. However, with a screened potential, the predicted mass for $\psi_{1}(2 D)$ is about $100 \mathrm{MeV}$ smaller than the observation. The $\psi_{1}(2 D)$ resonance can decay into $\chi_{c J}(1 P) \gamma$ and $\chi_{c J}(2 P) \gamma$ via the radiative transitions.

Considering $\psi(4160)$ as a pure $2^{3} D_{1}$ state, with the linear potential model, we predict that

$$
\begin{aligned}
& \Gamma\left[\psi(4160) \rightarrow \chi_{c 0}(1 P) \gamma\right] \simeq 150 \mathrm{keV}, \\
& \Gamma\left[\psi(4160) \rightarrow \chi_{c 1}(1 P) \gamma\right] \simeq 37 \mathrm{keV}, \\
& \Gamma\left[\psi(4160) \rightarrow \chi_{c 2}(1 P) \gamma\right] \simeq 17 \mathrm{keV} .
\end{aligned}
$$

Similar results are also obtained with the screened potential model. Our predictions of $\Gamma\left[\psi(4160) \rightarrow \chi_{c 0,1}(1 P) \gamma\right]$ are slightly smaller than those obtained in Ref. [17], however, our predictions are notablely larger than those in Ref. [8] (see Tab. IX]. Combining the measured decay width of $\psi(4160)$ with our predicted partial widths from the linear potential model, we estimate the branching fractions:

$$
\begin{aligned}
& \operatorname{Br}\left[\psi(4160) \rightarrow \chi_{c 0}(1 P) \gamma\right] \simeq 2.1 \times 10^{-3}, \\
& \operatorname{Br}\left[\psi(4160) \rightarrow \chi_{c 1}(1 P) \gamma\right] \simeq 0.5 \times 10^{-3}, \\
& \operatorname{Br}\left[\psi(4160) \rightarrow \chi_{c 2}(1 P) \gamma\right] \simeq 0.2 \times 10^{-3} .
\end{aligned}
$$

Our predictions are in the range of the recent measurements $\operatorname{Br}\left[\psi(4160) \rightarrow \chi_{c 1}(1 P) \gamma\right]<6.1 \times 10^{-3}$ and $\operatorname{Br}[\psi(4160) \rightarrow$ $\left.\chi_{c 2}(1 P) \gamma\right]<16.2 \times 10^{-3}$ from the Belle Collaboration [96]. We expect that more accurate observations can be carried out in future experiments. 
Furthermore, we calculate the partial decay width of $\Gamma\left[\psi(4160) \rightarrow \chi_{c J}(2 P) \gamma\right]$ with the linear and screened potential models, respectively. Our results are listed in Tab. IX Both of the models give a similar result. It is found that the decay rates of $\psi(4160) \rightarrow \chi_{c 0}(2 P) \gamma, \chi_{c 1}(2 P) \gamma$ are rater large, their partial decay widths may be $300 \sim 400 \mathrm{keV}$. Similar results were also obtained in Ref. [8, 10]. The estimated branching ratios are

$$
\begin{aligned}
& \operatorname{Br}\left[\psi(4160) \rightarrow \chi_{c 2}(2 P) \gamma\right] \simeq 0.3 \times 10^{-3}, \\
& \operatorname{Br}\left[\psi(4160) \rightarrow \chi_{c 1}(2 P) \gamma\right] \simeq 4.4 \times 10^{-3}, \\
& \operatorname{Br}\left[\psi(4160) \rightarrow \chi_{c 0}(2 P) \gamma\right] \simeq 4.4 \times 10^{-3} .
\end{aligned}
$$

It should be mentioned that $3 \mathrm{fb}^{-1}$ new data of $\psi(4160)$ have been collected at BESIII [99]. Using the cross section of $\sim 8$ nb based on BES and CLEO measurements [100-102], we estimate that $2.4 \times 10^{7}$ events of $\psi(4160)$ have been accumulated at BESIII. Thus, if $\psi(4160)$ is the $2^{3} D_{1}$ state indeed, it might provide us a source to look for the missing $\chi_{c 0}(2 P)$ and $\chi_{c 1}(2 P)$ states via the transition chains $\psi(4160) \rightarrow$ $\chi_{c J}(2 P) \gamma \rightarrow \psi(1 S, 2 S) \gamma \gamma$. The combined branching ratios of these decay chains, and the producing events of $\chi_{c J}(2 P)$ estimated by us have been listed in Tab. XII It is found that if the $\chi_{c J}(2 P)$ is to be observed at BESIII via the transition chains of $\psi(4160) \rightarrow \chi_{c J}(2 P) \gamma \rightarrow \psi(1 S, 2 S) \gamma \gamma \rightarrow \gamma \gamma \mu^{+} \mu^{-}$, one should accumulate more data samples of $\psi(4160)$ in the coming years.

The other three $2 D$-wave states, $\psi_{2}(2 D), \psi_{3}(2 D)$ and $\eta_{c 2}(2 D)$, are still not observed in experiments. With the masses and wavefuctions predicted from the linear and screened potential models, we calculate their radiative decay properties. Our results are listed in Tab. IX] It is seen that although the predictions in details from both linear and screened potential models have a notable difference, both models predict that these $2 D$ wave states $\psi_{2,3}(2 D)$ and $\eta_{c 2}(2 D)$ have relatively large transition rates into the $1 P$ - and $2 P$-wave states. The partial decay widths for the $2 D \rightarrow 1 P \gamma$ processes are about $10 \mathrm{~s} \mathrm{keV}$ and their branching ratios are estimated to be $B r[2 D \rightarrow 1 P \gamma] \sim O\left(10^{-4}\right)$; while the partial decay widths for the $2 D \rightarrow 2 P \gamma$ processes usually reach to $100 \mathrm{~s} \mathrm{keV}$ and their branching ratios are estimated to be $\operatorname{Br}[2 D \rightarrow 2 P \gamma] \sim$ $O\left(10^{-3}\right)$. The large decay rates of the $2 D \rightarrow 2 P \gamma$ processes were also predicted in Ref. [8]. We further estimate the combined branching ratios of the two-photon cascades $2 D \rightarrow n P \rightarrow m S$. Our results have been listed in Tab. XII In these decay chains, the most prominent two-photon cascades are $\psi_{2}(2 D) \rightarrow \chi_{c 1}(1 P) \gamma \rightarrow J / \psi \gamma \gamma \rightarrow \gamma \gamma \mu^{+} \mu^{-}(B r \simeq$ $\left.1.5 \times 10^{-5}\right)$, and $\eta_{c 2}(2 D) \rightarrow h_{c}(1 P) \gamma \rightarrow \eta_{c} \gamma \gamma \rightarrow \gamma \gamma K \bar{K} \pi$ $\left(B r \simeq 4.8 \times 10^{-5}\right)$. In coming years, Belle II will accumulate $10^{10} B \bar{B}$ data sample, which might let us obtain enough events of $2 D$-wave states via $B \rightarrow \psi_{2}(2 D) X$ and $B \rightarrow \eta_{c 2}(2 D) X$ decays. If the branching fractions of $\operatorname{Br}\left[B \rightarrow \psi_{2}(2 D) X\right]$ and $\operatorname{Br}\left[B \rightarrow \eta_{c 2}(2 D) X\right]$ are $O\left(10^{-5}\right)$, the missing $2 D$-wave states might be observed in the above two-photon cascades.

\section{6. $X(4140,4274)$ and the $3 P$ states}

Until now, no $3 P$ charmonium states have been established in experiments. According to the predicted masses and wave functions of the $3 P$ charmonium states, we estimate their radiative properties decay properties with both the linear and screened potential models, which are listed in Tab.VIII From the table, it is seen that most of our results from both models are similar in the magnitude. The $\chi_{c 0}(3 P)$ state has a large decay rate into the $\psi(3 S) \gamma$ channel, the partial width might be $10 \mathrm{~s} \sim 100 \mathrm{~s} \mathrm{keV}$, which is consistent with the prediction in Ref. [8]. The $\chi_{c 1,2}(3 P) / h_{c}(3 P)$ state has a large partial decay width into $\psi(1 S, 2 S, 3 S) \gamma / \eta_{c}(1 S, 2 S, 3 S) \gamma$ channels, which are $10 \mathrm{~s} \sim 100 \mathrm{~s} \mathrm{keV}$ as well. Combined the predicted widths from Ref. [8], the estimated branching ratios of $\operatorname{Br}\left[\chi_{c 1,2}(3 P) \rightarrow \psi(1 S, 2 S, 3 S) \gamma\right]$ and $\operatorname{Br}\left[\chi_{c 0}(3 P) \rightarrow \psi(3 S) \gamma\right]$ are $O\left(10^{-3}\right)$. Using $B \rightarrow \chi_{c 1,2}(3 P) K / h_{c}(3 P) K$ decays, the forthcoming Belle II and LHC experiments might reconstruct these higher $\chi_{c 1,2}(3 P) / h_{c}(3 P)$ states in the $\psi(1 S, 2 S) \gamma / \eta_{c}(2 S) \gamma$ decay modes.

Recently, two new charmonium-like states $X(4140)(\Gamma \simeq 16$ $\mathrm{MeV})$ and $X(4274)(\Gamma \simeq 56 \mathrm{MeV})$ are confirmed by the $\mathrm{LHCb}$ collaboration [52]. Their quantum numbers are determined to be $J^{P C}=1^{++}$. According to the predicted mass from the linear potential model, the $X(4274)$ might be a good candidate of $\chi_{c 1}(3 P)$. However, within the screened potential model, $X(4140)$ seems to favor the $\chi_{c 1}(3 P)$ state. If the $X(4140)$ state is assigned as $\chi_{c 1}(3 P)$, within the screened potential model the partial radiative decay widths of the dominant channels are predicted to be

$$
\begin{aligned}
\Gamma[\psi(4140) \rightarrow J / \psi \gamma] & \simeq 38 \mathrm{keV}, \\
\Gamma[\psi(4140) \rightarrow \psi(2 S) \gamma] & \simeq 51 \mathrm{keV}, \\
\Gamma[\psi(4140) \rightarrow \psi(3 S) \gamma] & \simeq 36 \mathrm{keV},
\end{aligned}
$$

Combined the average measured width with the predicted partial radiative decay widths of $X(4140)$, the branching ratios are estimated to be

$$
\begin{aligned}
\operatorname{Br}[\psi(4140) \rightarrow J / \psi \gamma] & \simeq 2.4 \times 10^{-3}, \\
\operatorname{Br}[\psi(4140) \rightarrow \psi(2 S) \gamma] & \simeq 3.2 \times 10^{-3}, \\
\operatorname{Br}[\psi(4140) \rightarrow \psi(3 S) \gamma] & \simeq 2.3 \times 10^{-3} .
\end{aligned}
$$

While, if the $X(4274)$ state is assigned as $\chi_{c 1}(3 P)$, within the linear potential model the partial radiative decay widths of the dominant channels are predicted to be

$$
\begin{aligned}
\Gamma[\psi(4274) \rightarrow J / \psi \gamma] & \simeq 48 \mathrm{keV}, \\
\Gamma[\psi(4274) \rightarrow \psi(2 S) \gamma] & \simeq 88 \mathrm{keV}, \\
\Gamma[\psi(4274) \rightarrow \psi(3 S) \gamma] & \simeq 297 \mathrm{keV},
\end{aligned}
$$

Combined the measured width with the predicted partial radiative decay widths of $X(4274)$, the branching ratios are estimated to be

$$
\begin{aligned}
\operatorname{Br}[\psi(4274) \rightarrow J / \psi \gamma] & \simeq 0.9 \times 10^{-3}, \\
\operatorname{Br}[\psi(4274) \rightarrow \psi(2 S) \gamma] & \simeq 1.6 \times 10^{-3}, \\
\operatorname{Br}[\psi(4274) \rightarrow \psi(3 S) \gamma] & \simeq 5.3 \times 10^{-3} .
\end{aligned}
$$

The search for $X(4274)$ and $X(4140)$ in the $\psi(1 S, 2 S, 3 S) \gamma$ channels and the measurements of their partial width ratios might be helpful to uncover the nature of these two newly observed states. 


\section{7. $4 S$ states}

In the $4 S$ states, the $\psi(4 S)$ resonance seems to favor the $1^{--}$state $\psi(4415)$ according to the linear potential model predictions [8]. However, there are other explanations about $\psi(4415)$. For example in the screened potential model, $\psi(4415)$ more favors $\psi(5 S)$ other than $\psi(4 S)$ [11], while with a coupled-channel method the $\psi(4415)$ resonance are suggested to be the $\psi_{1}(1 D)$ resonance [17]. According to the screened potential model prediction, the $J^{P C}=1^{--}$state $X(4260)$ from the PDG [6] could be a good candidate of the $\psi(4 S)$. Very recently, BESIII Collaboration observed a new structure $Y(4220)$ with a width of $\Gamma \simeq 66 \mathrm{MeV}$ in the $e^{+} e^{-} \rightarrow \pi^{+} \pi^{-} h_{c}$ cross sections [97]. The resonance parameters of $Y(4220)$ are consistent with those of the resonance observed in the $e^{+} e^{-} \rightarrow \omega \chi_{c 0}$ [98]. The newly observed $Y(4220)$ might be a candidate of $\psi(4 S)$ as well. To establish the $4 S$ states, more studies are needed.

With the screened potential model we predict that the masses of the $4 S$ states are about $4.28 \mathrm{GeV}$, while in the linear potential model their masses are about $4.41 \mathrm{GeV}$. Taking the predicted masses of $\psi(4 S)$ with $4412 \mathrm{MeV}$ and $4281 \mathrm{GeV}$ from the linear and screened potential models, respectively, we calculate the radiative transitions of the $\psi(4 S)$ state within these two models. Our results are listed in Tab.VII It is found that both the linear and screened potential models give comparable predictions of the decay rates for the $4 S$ states in the magnitude, although the details are different. The decay rates of $4 S \rightarrow 2 P, 3 P$ are sizeable, the partial widths for the transitions $\psi(4 S) \rightarrow \chi_{c J}(2 P) \gamma$ are about $10 \sim 20 \mathrm{keV}$, and for the transitions $\psi(4 S) \rightarrow \chi_{c J}(3 P) \gamma$ are about $20 \sim 80 \mathrm{keV}$. Combined the predicted widths $\Gamma \simeq 78 \mathrm{MeV}$ from Ref. [8], the branching ratios for the $4 S \rightarrow 2 P, 3 P$ transitions are $O\left(10^{-4}\right)$.

In coming years, BESIII plan to collect $5 \sim 10 \mathrm{fb}^{-1}$ data samples at $\psi(4 S)$ [99]. Using the cross section of $\sim 4 \mathrm{nb}$ based on BES measurements [100, 101], we expect to accumulate $(2 \sim 4) \times 10^{7} \psi(4 S)$ 's. To know the production possibilities of $2 P$ and $3 P$ states via the radiative decay of $\psi(4 S)$, we estimate the number of production events from the two-photon cascades $\psi(4 S) \rightarrow \chi_{c J}(2 P, 3 P) \gamma \rightarrow \psi(1 S, 2 S) \gamma \gamma$, which has been listed in Tab. XIII. Unfortunately, it is found that the higher $2 P$ and $3 P$ states are not able to be produced via these radiative decay chains at BESIII.

\section{Higher multipole contributions}

In our calculations, we find that the corrections from the magnetic part to some radiative transitions of the $S$-, $P$ - and $D$-wave states are notable (see Tabs VIIX). For example the magnetic part could give a $10-30 \%$ correction to the radiative partial decay widths of $\Gamma\left[\chi_{c J}(1 P) \rightarrow J / \psi \gamma\right], \Gamma\left[\psi_{1}(1 D) \rightarrow\right.$ $\left.\chi_{c 1,2}(1 P) \gamma\right]$ and $\Gamma\left[\psi(3 S) \rightarrow \chi_{c J}(1 P) \gamma\right]$. This large correction is mainly caused by the interferences between the "extra" electric-dipole term $E_{R}$ from the magnetic part and the leading E1 transitions. About the higher order EM corrections to the radiative transitions, some discussions can be found in the literature 19, 20, 74, 103 109].
In experiments, the higher order amplitudes for the transitions $\chi_{c 1,2}(1 P) \rightarrow J / \psi \gamma$ and/or $\psi(2 S) \rightarrow \chi_{c 1.2}(1 P) \gamma$ have been measured in different experiments [110-115]. Our predictions with both the linear and screened potential models compared with the data have been listed in Tab. XIV From the table, it is seen that both models give comparable results. The predicted ratios between the magnetic quadrupole amplitude and the electric-dipole amplitude, $a_{2} / a_{1}$, for the $\chi_{c 1,2}(1 P) \rightarrow J / \psi \gamma$ processes are in good agreement with the recent measurements from CLEO [114]. The ratios of $a_{2} / a_{1}$ for the $\psi(2 S) \rightarrow \chi_{c 1,2}(1 P) \gamma$ are small. Their absolute values are comparable to the measurements from CLEO [114], however, the sign of $a_{2} / a_{1}$ predicted by us seems to be opposite to the measurements. It should be mentioned that our prediction of $a_{2} / a_{1}$ for the $\psi(2 S) \rightarrow \chi_{c 2}(1 P) \gamma$ is consistent with the previous measurement from BESII [113]. More accurate measurements may clarify the sign problem. The ratios between the "extra" electric-dipole amplitudes $E_{R}$ and the $a_{1}$ are also predicted. It is found that $\left|\frac{E_{R}}{a_{1}}\right| \simeq\left|\frac{a_{2}}{a_{1}}\right|$.

Furthermore, we predict the ratios $E_{R} / a_{1}$ and $a_{2} / a_{1}$ for some unmeasured processes $\psi_{1}(1 D) \rightarrow \chi_{c 1,2}(1 P) \gamma$ and $\chi_{c J}(n P) \rightarrow \psi(m S) \gamma$, in which the magnetic part plays an important role. Our results have been listed in Tab. XV From the table, it found that most of the ratios are fairly large. Some ratios can reach to $\sim 30 \%$. Since the $\psi_{1}(1 D)$ and $\chi_{c 2}(2 P)$ have been established, and the ratios of $a_{2} / a_{1}$ for $\psi_{1}(1 D) \rightarrow \chi_{c 1,2}(1 P) \gamma$ and $\chi_{c 2}(2 P) \rightarrow J \psi \gamma$ are fairly large, we suggest the experimentalists measure the ratios of $a_{2} / a_{1}$ for these transitions in future experiments.

\section{SUMMARY}

In this work we calculate the charmonium spectrum with two models, linear potential model and screened potential model. We should emphasize that (i) the hyperfine and fine splittings show less model dependence. The predicted splitting, $m\left(2^{3} P_{2}\right)-m\left(2^{3} P_{1}\right) \simeq 90 \mathrm{MeV}$, does not support the $X(3915)$ assigned as the $\chi_{c 0}(2 P)$ state. (ii) In the screened potential model, the states $X(4260)$ and $X(4360)$ with $J^{P C}=1^{--}$ may be good candidates of the $\psi(4 S)$ and $\psi_{1}(3 D)$, respectively. (iii) For the newly confirmed $J^{P C}=1^{++}$states $X(4140)$ and $X(4274)$ by the $\mathrm{LHCb}$, within the linear potential model the $X(4274)$ might be identified as the $\chi_{c 1}(3 P)$ states. While within the screened potential model, the $X(4140)$ is a good candidate of $\chi_{c 1}(3 P)$.

Second, we further evaluate the EM transitions of charmonium states up to the $4 S$ multiplet. It is found that (i) for the EM transitions of the well-established low-lying charmonium states $J / \psi, \psi(2 S), \chi_{c J}(1 P), h_{c}(1 P)$ and $\psi(3770)$, both linear potential and screened potential models give similar descriptions, which are in reasonable agreement with the measurements. (ii) Identifying the newly observed state $X(3823)$ at Belle and BESIII as the $\psi_{2}(1 D)$, its EM decay properties of are in good agreement with the measurements. (iii) Assigning the $X(3872)$ resonance as the $\chi_{c 1}(2 P)$ state, the ratio $\frac{\Gamma[X(3872) \rightarrow \psi(2 S) \gamma]}{\Gamma[X(3872) \rightarrow J / \psi \gamma]} \simeq 1.3$ predicted by us is close to the lower limit of the measurements from the BaBar and LHCb. Thus 
the $X(3872)$ as the $\chi_{c 1}(2 P)$ can not be excluded.

Thirdly, we discuss the observations of the missing charmonium states by using radiative transitions. (i) The large $B \bar{B}$ data sample from Belle II should let us have chances to establish the missing $\eta_{c 2}(1 D)$ and $\psi_{3}(1 D)$ states in forthcoming experiments. The $\eta_{c 2}(1 D)$ state should be produced via the $B \rightarrow \eta_{c 2}(1 D) K$ process and reconstructed in the $h_{c}(1 P) \gamma$ decay mode with $h_{c}(1 P) \rightarrow \eta_{c} \gamma$ and $\eta_{c} \rightarrow K \bar{K} \pi$. While the $\psi_{3}(1 D)$ state should be produced via the $B \rightarrow \psi_{3}(1 D) K$ process, and reconstructed in the $\chi_{c 2}(1 P) \gamma$ decay mode with $\chi_{c 2}(1 P) \rightarrow J / \psi \gamma$ and $J / \psi \rightarrow \mu^{+} \mu^{-} / e^{+} e^{-}$. (ii) If BESIII can accumulate $5 \sim 10 \mathrm{fb}^{-1} \psi(4040)$ data sample in the coming years, significant numbers of $\chi_{c 2}(2 P)$ is to be produced via the radiative decay of $\psi(4040)$, and reconstructed in the $J / \psi \gamma$ decay mode with $J / \psi \rightarrow \mu^{+} \mu^{-}$. (iii) Relatively large data samples of $2 D$-wave states $\psi_{2}(2 D)$ and $\eta_{c 2}(2 D)$ might be collected at Belle II or LHCb via $B \rightarrow \psi_{2}(2 D) X$ and $B \rightarrow \eta_{c 2}(2 D) X$ decays in forthcoming experiments, the twophoton decay chains $2^{3} D_{2} \rightarrow \chi_{c 1}(1 P) \gamma \rightarrow J / \psi \gamma \gamma \rightarrow \gamma \gamma \mu^{+} \mu^{-}$ $\left(B r \simeq 1.5 \times 10^{-5}\right)$, and $\eta_{c 2}(2 D) \rightarrow h_{c}(1 P) \gamma \rightarrow \eta_{c}(1 S) \gamma \gamma \rightarrow$ $K \bar{K} \pi \gamma \gamma\left(B r \simeq 4.8 \times 10^{-5}\right)$ are worth observing. (iv) The missing $3 P$-wave states might be observed at LHCb and Belle II in the $B \rightarrow \chi_{c 1,2}(3 P) K / h_{c}(3 P) K$ decays, and reconstructed in the $\psi(1 S, 2 S) \gamma / \eta_{c}(2 S) \gamma$ decay modes with $\psi(1 S, 2 S) \rightarrow$ $\mu^{+} \mu^{-} / \eta_{c}(2 S) \rightarrow K \bar{K} \pi$.

Finally, we study the corrections of higher EM multipole amplitudes to the EM transitions. The magnetic part could give about a $10 \sim 30 \%$ correction to the radiative partial decay widths of $\Gamma\left[\chi_{c J}(1 P) \rightarrow J / \psi \gamma\right], \Gamma\left[\psi_{1}(1 D) \rightarrow \chi_{c 1,2}(1 P) \gamma\right]$ and $\Gamma\left[\psi(3 S) \rightarrow \chi_{c J}(1 P) \gamma\right]$. This large correction is mainly caused by the interferences between the "extra" electric-dipole term $E_{R}$ from the magnetic part and the leading E1 amplitudes. Our predictions for the normalized magnetic quadrupole amplitude $M_{2}$ of the $\chi_{c 1,2}(1 P) \rightarrow J / \psi \gamma$ processes are in good agreement with the recent measurements from CLEO [114]. About the normalized magnetic quadrupole amplitude of $\psi(2 S) \rightarrow$ $\chi_{c 1,2}(1 P) \gamma$, there may be a sign difference between our predictions and the measurements. The normalized "extra" electricdipole amplitudes $E_{R}$ are also predicted. It is found that $\left|E_{R}\right| \simeq\left|M_{2}\right|$. Furthermore, we find that there are fairly large magnetic quadrupole amplitudes $M_{2}$ for the $\chi_{c 1,2}(2 P, 3 P) \rightarrow$ $\psi(1 S, 2 S) \gamma$ and $\psi_{1}(1 D) \rightarrow \chi_{c 1,2}(1 P)$ processes. We suggest the experimentalists measure the higher magnetic quadrupole amplitudes $M_{2}$ of the $\chi_{2}(2 P) \rightarrow \psi(1 S, 2 S) \gamma$ and $\psi_{1}(1 D) \rightarrow$ $\chi_{c 1,2}(1 P)$ processes in future experiments.

\section{Acknowledgement}

We thank Shi-Lin Zhu and Qiang Zhao for helpful comments and suggestions. This work is supported, in part, by the National Natural Science Foundation of China (Grants No. 11075051, No. 11375061, and No. 11405053), and the Hunan Provincial Natural Science Foundation (Grant No. 13JJ1018).
[1] E. Eichten, S. Godfrey, H. Mahlke and J. L. Rosner, Quarkonia and their transitions, Rev. Mod. Phys. 80, 1161 (2008).

[2] A. J. Bevan et al. [BaBar and Belle Collaborations], The Physics of the $B$ Factories, Eur. Phys. J. C 74, 3026 (2014).

[3] N. Brambilla et al., Heavy quarkonium: progress, puzzles, and opportunities, Eur. Phys. J. C 71, 1534 (2011).

[4] M. B. Voloshin, Charmonium, Prog. Part. Nucl. Phys. 61, 455 (2008).

[5] H. X. Chen, W. Chen, X. Liu and S. L. Zhu, The hidden-charm pentaquark and tetraquark states, Phys. Rept. 639, 1 (2016).

[6] K. A. Olive et al. [Particle Data Group Collaboration], Review of Particle Physics, Chin. Phys. C 38, 090001 (2014).

[7] S. Godfrey and N. Isgur, Mesons in a Relativized Quark Model with Chromodynamics, Phys. Rev. D 32, 189 (1985).

[8] T. Barnes, S. Godfrey and E. S. Swanson, Higher charmonia, Phys. Rev. D 72, 054026 (2005).

[9] S. Godfrey and K. Moats, Bottomonium Mesons and Strategies for their Observation, Phys. Rev. D 92, 054034 (2015).

[10] B. Q. Li, C. Meng and K. T. Chao, Search for $\chi_{c_{J}}(2 P)$ from Higher Charmonim E1 Transitions and X,Y,Z States, arXiv:1201.4155 [hep-ph].

[11] B. Q. Li and K. T. Chao, Higher Charmonia and X,Y,Z states with Screened Potential, Phys. Rev. D 79, 094004 (2009).

[12] E. Eichten, K. Gottfried, T. Kinoshita, K. D. Lane and T. M. Yan, Charmonium: Comparison with Experiment, Phys. Rev. D 21, 203 (1980).

[13] E. J. Eichten, K. Lane and C. Quigg, B meson gateways to missing charmonium levels, Phys. Rev. Lett. 89, 162002 (2002).
[14] T. Barnes and S. Godfrey, Charmonium options for the X(3872), Phys. Rev. D 69, 054008 (2004).

[15] K.T. Chao and J.H. Liu, in Proceedings of the Workshop on Weak Interactions and CP Violation, Beijing, August 22-26, 1989, edited by T. Huang and D.D. Wu, World Scientific (Singapore, 1990) p.109-p.117.

[16] Y. B. Ding, K. T. Chao and D. H. Qin, Screened $Q \bar{Q}$ potential and spectrum of heavy quarkonium, Chin. Phys. Lett.10, 460 (1993).

[17] J. Segovia, A. M. Yasser, D. R. Entem and F. Fernandez, $J^{P C}=1^{--}$hidden charm resonances, Phys. Rev. D 78, 114033 (2008).

[18] L. Cao, Y. C. Yang and H. Chen, Charmonium states in QCD-inspired quark potential model using Gaussian expansion method, Few Body Syst. 53, 327 (2012).

[19] J. J. Dudek, R. G. Edwards and D. G. Richards, Radiative transitions in charmonium from lattice QCD, Phys. Rev. D 73, 074507 (2006).

[20] J. J. Dudek, R. Edwards and C. E. Thomas, Exotic and excitedstate radiative transitions in charmonium from lattice QCD, Phys. Rev. D 79, 094504 (2009).

[21] D. Becirevi, M. Kruse and F. Sanfilippo, Lattice QCD estimate of the $\eta_{c}(2 S) \rightarrow J / \psi \gamma$ decay rate, JHEP 1505, 014 (2015).

[22] G. C. Donald, C. T. H. Davies, R. J. Dowdall, E. Follana, K. Hornbostel, J. Koponen, G. P. Lepage and C. McNeile, Precision tests of the $J / \psi$ from full lattice QCD: mass, leptonic width and radiative decay rate to $\eta_{c}$, Phys. Rev. D 86, 094501 (2012).

[23] Y. Chen et al., Radiative transitions in charmonium from $N_{f}=$ 
TABLE V: Partial widths (keV) of the M1 radiative transitions for some low-lying $S$-wave charmonium states. LP and SP stand for our results obtained from the linear potential and screened potential models, respectively. For comparison, the predictions from the relativistic quark model [37], NR and GI models [8] are listed in the table as well. The experimental average data are taken from the PDG [6].

\begin{tabular}{|c|c|c|c|c|c|c|c|c|c|c|c|}
\hline \multirow{2}{*}{$\begin{array}{c}\text { Initial } \\
\text { state } \\
\end{array}$} & \multirow{2}{*}{$\begin{array}{l}\text { Final } \\
\text { state }\end{array}$} & \multicolumn{4}{|c|}{$E_{\gamma}(\mathrm{MeV})$} & \multicolumn{5}{|c|}{$\Gamma_{\mathrm{M} 1}(\mathrm{keV})$} & \multirow{2}{*}{$\frac{\Gamma_{\mathrm{M} 1}(\mathrm{keV})}{\text { Exp. }}$} \\
\hline & & [37] & $\mathrm{NR}[8]$ & GI [8] & Ours & [37] & $N R[8]$ & GI[8] & LP & SP & \\
\hline$J / \psi$ & $\eta_{c}(1 S)$ & 115 & 116 & 115 & 111 & 1.05 & 2.9 & 2.4 & 2.39 & 2.44 & $1.58 \pm 0.37$ \\
\hline \multirow[t]{2}{*}{$\psi(2 S)$} & $\eta_{c}(2 S)$ & 32 & 48 & 48 & 47 & 0.043 & 0.21 & 0.17 & 0.19 & 0.19 & \multirow{3}{*}{$\begin{array}{l}0.21 \pm 0.15 \\
1.24 \pm 0.29\end{array}$} \\
\hline & $\eta_{c}(1 S)$ & 639 & 639 & 638 & 635 & 0.95 & 4.6 & 9.6 & 8.08 & 7.80 & \\
\hline$\eta_{c}(2 S)$ & $J / \psi$ & 514 & 501 & 501 & 502 & 1.53 & 7.9 & 5.6 & 2.64 & 2.29 & \\
\hline \multirow[t]{3}{*}{$\psi(3 S)$} & $\eta_{c}(3 S)$ & & 29 & 35 & $30 / 36$ & & 0.046 & 0.067 & 0.051 & 0.088 & \\
\hline & $\eta_{c}(2 S)$ & & 382 & 436 & 381 & & 0.61 & 2.6 & 1.65 & 1.78 & \\
\hline & $\eta_{c}(1 S)$ & & 922 & 967 & 918 & & 3.5 & 9.0 & 6.66 & 6.76 & \\
\hline
\end{tabular}

TABLE VI: Partial widths $\Gamma(\mathrm{keV})$ and branching ratios $B r$ for the radiative transitions (E1 dominant) between the low-lying charmonium states. LP and SP stand for our results obtained from the linear potential and screened potential models, respectively. For comparison, the predictions from the relativistic quark model [37], NR and GI models [8] and SNR model [11] are listed in the table as well. The experimental average data are taken from the PDG. $\Gamma_{\mathrm{E} 1}$ and $\Gamma_{\mathrm{EM}}$ stands for the E1 and EM transition widths, respectively.

\begin{tabular}{|c|c|c|c|c|c|c|c|c|c|c|c|c|c|}
\hline \multirow{2}{*}{$\begin{array}{c}\text { Initial } \\
\text { state }\end{array}$} & \multirow{2}{*}{$\begin{array}{l}\text { Final } \\
\text { state }\end{array}$} & \multirow{2}{*}{$\frac{E_{\gamma}(\mathrm{MeV})}{\text { Ours }}$} & \multicolumn{5}{|c|}{$\underline{\Gamma_{\mathrm{E} 1}(\mathrm{keV})}$} & \multicolumn{3}{|c|}{ 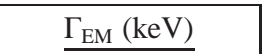 } & \multicolumn{3}{|c|}{$B r(\%)$} \\
\hline & & & [37] & NR/GI [8] & $\mathrm{SNR}_{0 / 1}[11]$ & LP & SP & LP & SP & Exp. & LP & SP & Exp. \\
\hline \multirow[t]{3}{*}{$\psi(2 S)$} & $\chi_{c 2}(1 P)$ & 128 & 18.2 & $38 / 24$ & $43 / 34$ & 36 & 44 & 38 & 46 & $25.2 \pm 2.9$ & 13.3 & 15.7 & $9.1 \pm 0.3$ \\
\hline & $\chi_{c 1}(1 P)$ & 171 & 22.9 & $54 / 29$ & $62 / 36$ & 45 & 48 & 42 & 45 & $25.5 \pm 2.8$ & 14.7 & 15.7 & $9.6 \pm 0.3$ \\
\hline & $\chi_{c 0}(1 P)$ & 261 & 26.3 & $63 / 26$ & $74 / 25$ & 27 & 26 & 22 & 22 & $26.3 \pm 2.6$ & 7.7 & 7.7 & $10.0 \pm 0.3$ \\
\hline$\eta_{c}(2 S)$ & $h_{c}(1 P)$ & 112 & 41 & 49 / 36 & $146 / 104$ & 49 & 52 & 49 & 52 & & 0.43 & 0.46 & \\
\hline$\chi_{c 2}(1 P)$ & $J / \psi$ & 429 & 327 & $424 / 313$ & $473 / 309$ & 327 & 338 & 284 & 292 & 371 & 14.6 & 15.0 & $19.2 \pm 0.7$ \\
\hline$\chi_{c 1}(1 P)$ & & 390 & 265 & 314 / 239 & 244 & 269 & 278 & 306 & 319 & $285 \pm 14$ & 34.8 & 36.3 & $33.9 \pm 1.2$ \\
\hline$\chi_{c 0}(1 P)$ & & 303 & 121 & $152 / 114$ & 117 & 141 & 146 & 172 & 179 & $133 \pm 8$ & 1.6 & 1.7 & $1.3 \pm 0.1$ \\
\hline$h_{c}(1 P)$ & $\eta_{c}(1 S)$ & 499 & 560 & 498 / 352 & $764 / 323$ & 361 & 373 & 361 & 373 & $357 \pm 280$ & 51.6 & 51.0 & $51.0 \pm 6.0$ \\
\hline \multirow[t]{3}{*}{$\psi_{1}(1 D)$} & $\chi_{c 2}(1 P)$ & 215 & 6.9 & $4.9 / 3.3$ & $5.8 / 4.6$ & 5.4 & 5.7 & 7.1 & 8.1 & $<24.8$ & $4.8 \times 10^{-2}$ & $5.3 \times 10^{-2}$ & $<9.0 \times 10^{-2}$ \\
\hline & $\chi_{c 1}(1 P)$ & 258 & 135 & $125 / 77$ & $150 / 93$ & 115 & 111 & 138 & 135 & $81 \pm 27$ & 0.55 & 0.58 & $0.29 \pm 0.06$ \\
\hline & $\chi_{c 0}(1 P)$ & 346 & 355 & $403 / 213$ & $486 / 197$ & 243 & 232 & 272 & 261 & $202 \pm 42$ & 0.99 & 0.95 & $0.73 \pm 0.09$ \\
\hline \multirow[t]{2}{*}{$\psi_{2}(1 D)$} & $\chi_{c 2}(1 P)$ & 258 & 59 & $64 / 66$ & $70 / 55$ & 79 & 82 & 91 & 96 & & 13.3 & 14.1 & \\
\hline & $\chi_{c 1}(1 P)$ & 299 & 215 & $307 / 268$ & $342 / 208$ & 281 & 291 & 285 & 296 & & 41.9 & 43.5 & \\
\hline
\end{tabular}

2 twisted mass lattice QCD, Phys. Rev. D 84, 034503 (2011).

[24] Y. B. Yang et al. [CLQCD Collaboration], Lattice study on $\eta_{c 2}$ and X(3872), Phys. Rev. D 87, 014501 (2013).

[25] L. Liu et al. [Hadron Spectrum Collaboration], Excited and exotic charmonium spectroscopy from lattice QCD, JHEP 1207, 126 (2012).

[26] D. Becirevic and F. Sanfilippo, Lattice QCD study of the radiative decays $J / \psi \rightarrow \eta_{c} \gamma$ and $h_{c} \rightarrow \eta_{c} \gamma$, JHEP 1301, 028 (2013).

[27] A. Y. Khodjamirian, Dispersion Sum Rules for the Amplitudes of Radiative Transitions in Quarkonium, Phys. Lett. B 90, 460 (1980).

[28] V. A. Beilin and A. V. Radyushkin, Quantum Chromodynamic Sum Rules and $J / \psi \rightarrow \eta_{c} \gamma$ Decay, Nucl. Phys. B 260, 61 (1985).

[29] S. L. Zhu and Y. B. Dai, Radiative decays of heavy hadrons from light cone QCD sum rules in the leading order of HQET, Phys. Rev. D 59, 114015 (1999).

[30] E. J. Eichten, K. Lane and C. Quigg, Charmonium levels near threshold and the narrow state $X(3872) \rightarrow \pi^{+} \pi^{-} J / \psi$, Phys. Rev. D 69, 094019 (2004).

[31] F. De Fazio, Radiative transitions of heavy quarkonium states,
Phys. Rev. D 79, 054015 (2009) [Phys. Rev. D 83, 099901 (2011)].

[32] Z. G. Wang, Analysis of the radiative decays among the charmonium states, Int. J. Theor. Phys. 51, 1518 (2012).

[33] N. Brambilla, Y. Jia and A. Vairo, Model-independent study of magnetic dipole transitions in quarkonium, Phys. Rev. D 73, 054005 (2006)

[34] Y. Jia, W. L. Sang and J. Xu, Is the $J^{P}=2^{-}$assignment for the $X(3872)$ compatible with the radiative transition data?, arXiv:1007.4541 [hep-ph].

[35] N. Brambilla, P. Pietrulewicz and A. Vairo, Modelindependent Study of Electric Dipole Transitions in Quarkonium, Phys. Rev. D 85, 094005 (2012).

[36] A. Pineda and J. Segovia, Improved determination of heavy quarkonium magnetic dipole transitions in potential nonrelativistic QCD, Phys. Rev. D 87, 074024 (2013).

[37] D. Ebert, R. N. Faustov and V. O. Galkin, Properties of heavy quarkonia and $B_{c}$ mesons in the relativistic quark model, Phys. Rev. D 67, 014027 (2003).

[38] T. H. Wang and G. L. Wang, Radiative E1 decays of $X(3872)$, Phys. Lett. B 697, 233 (2011).

[39] H. W. Ke, X. Q. Li and Y. L. Shi, The radiative decays of $0^{++}$ 
TABLE VII: Partial widths $\Gamma(\mathrm{keV})$ and branching ratios $B r$ for the radiative transitions of the higher $S$-wave states. LP and SP stand for our results obtained from the linear potential and screened potential models, respectively. For comparison, the predictions from the NR and GI models [8] are listed in the table as well.

\begin{tabular}{|c|c|c|c|c|c|c|c|c|c|c|c|c|c|}
\hline \multirow{2}{*}{$\begin{array}{c}\text { Initial } \\
\text { state }\end{array}$} & \multirow{2}{*}{$\begin{array}{c}\text { Final } \\
\text { state }\end{array}$} & \multicolumn{4}{|c|}{$E_{\gamma}(\mathrm{MeV})$} & \multicolumn{4}{|c|}{$\Gamma_{\mathrm{E} 1}(\mathrm{keV})$} & \multicolumn{2}{|c|}{$\Gamma_{\mathrm{EM}}(\mathrm{keV})$} & \multicolumn{2}{|c|}{$\underline{B r}$} \\
\hline & & NR [8] & GI [8] & LP & SP & NR [8] & GI [8] & LP & SP & LP & SP & LP & SP \\
\hline$\psi(3 S)$ & $\chi_{c 2}(2 P)$ & 67 & 119 & 111 & 111 & 14 & 48 & 65 & 79 & 67 & 82 & $8.4 \times 10^{-4}$ & $1.0 \times 10^{-3}$ \\
\hline \multirow[t]{5}{*}{$80 \pm 10^{a}$} & $\chi_{c 1}(2 P)$ & 113 & 145 & 138 & 138 & 39 & 43 & 58 & 71 & 55 & 67 & $6.9 \times 10^{-4}$ & $8.4 \times 10^{-4}$ \\
\hline & $\chi_{c 0}(2 P)$ & 184 & 180 & 167 & 187 & 54 & 22 & 21 & 31 & 19 & 27 & $2.4 \times 10^{-4}$ & $3.4 \times 10^{-4}$ \\
\hline & $\chi_{c 2}(1 P)$ & 455 & 508 & 455 & 455 & 0.7 & 13 & 0.21 & 2.1 & 0.25 & 2.5 & $3.1 \times 10^{-6}$ & $3.1 \times 10^{-5}$ \\
\hline & $\chi_{c 1}(1 P)$ & 494 & 547 & 494 & 494 & 0.53 & 0.85 & 4.8 & 8.0 & 4.0 & 6.7 & $5.0 \times 10^{-5}$ & $8.4 \times 10^{-5}$ \\
\hline & $\chi_{c 0}(1 P)$ & 577 & 628 & 577 & 577 & 0.27 & 0.63 & 9.1 & 10.6 & 5.9 & 6.7 & $7.4 \times 10^{-5}$ & $8.4 \times 10^{-5}$ \\
\hline$\eta_{c}(3 S)$ & $h_{c}(2 P)$ & 108 & 108 & 108 & 108 & 105 & 64 & 104 & 128 & 104 & 128 & $1.3 \times 10^{-3}$ & $1.6 \times 10^{-3}$ \\
\hline $80^{b}$ & $h_{c}(1 P)$ & 485 & 511 & 456 & 456 & 9.1 & 28 & 0.045 & 1.4 & 0.045 & 1.4 & $5.6 \times 10^{-7}$ & $1.8 \times 10^{-5}$ \\
\hline$\psi(4 S)$ & $\chi_{c 2}(1 P)$ & 775 & 804 & 773 & 664 & 0.61 & 5.2 & 0.13 & 0.66 & 0.17 & 0.84 & $2.2 \times 10^{-6}$ & $1.1 \times 10^{-5}$ \\
\hline \multirow[t]{8}{*}{$78^{b}$} & $\chi_{c 1}(1 P)$ & 811 & 841 & 809 & 701 & 0.41 & 0.53 & 3.8 & 3.9 & 2.9 & 3.0 & $3.7 \times 10^{-5}$ & $3.8 \times 10^{-5}$ \\
\hline & $\chi_{c 0}(1 P)$ & 887 & 915 & 884 & 778 & 0.18 & 0.13 & 7.5 & 6.2 & 3.7 & 2.7 & $4.7 \times 10^{-5}$ & $4.2 \times 10^{-5}$ \\
\hline & $\chi_{c 2}(2 P)$ & 421 & 446 & 458 & 339 & 0.62 & 15 & 11 & 4.7 & 13 & 5.3 & $1.7 \times 10^{-4}$ & $6.8 \times 10^{-5}$ \\
\hline & $\chi_{c 1}(2 P)$ & 423 & 469 & 482 & 364 & 0.49 & 0.92 & 24 & 12 & 20 & 11 & $2.6 \times 10^{-4}$ & $1.4 \times 10^{-4}$ \\
\hline & $\chi_{c 0}(2 P)$ & 527 & 502 & 510 & 411 & 0.24 & 0.39 & 17 & 12 & 12 & 8.7 & $1.5 \times 10^{-4}$ & $1.1 \times 10^{-4}$ \\
\hline & $\chi_{c 2}(3 P)$ & 97 & 112 & 101 & 69 & 68 & 66 & 80 & 39 & 82 & 40 & $1.1 \times 10^{-3}$ & $5.1 \times 10^{-4}$ \\
\hline & $\chi_{c 1}(3 P)$ & 142 & 131 & 126 & 88 & 126 & 54 & 74 & 38 & 71 & 37 & $9.1 \times 10^{-4}$ & $4.7 \times 10^{-4}$ \\
\hline & $\chi_{c 0}(3 P)$ & 208 & 155 & 178 & 133 & 0.003 & 25 & 40 & 23 & 36 & 21 & $4.6 \times 10^{-4}$ & $2.7 \times 10^{-4}$ \\
\hline$\eta_{c}(4 S)$ & $h_{c}(1 P)$ & 782 & 808 & 778 & 675 & 5.2 & 9.6 & 0.29 & 0.63 & 0.29 & 0.63 & $4.8 \times 10^{-6}$ & $1.0 \times 10^{-5}$ \\
\hline \multirow[t]{2}{*}{$61^{b}$} & $h_{c}(2 P)$ & 427 & 444 & 461 & 348 & 10.1 & 31.3 & 20 & 7.9 & 20 & 7.9 & $3.3 \times 10^{-4}$ & $1.3 \times 10^{-4}$ \\
\hline & $h_{c}(3 P)$ & 104 & 106 & 142 & 70 & 159 & 101 & 102 & 70 & 102 & 70 & $1.7 \times 10^{-3}$ & $1.1 \times 10^{-3}$ \\
\hline
\end{tabular}

${ }^{a}$ Width (MeV) from the PDG [6].

${ }^{b}$ Predicted width $(\mathrm{MeV})$ from Ref. [8].

and $1^{+-}$heavy mesons, Phys. Rev. D 87, 054022 (2013).

[40] P. Guo, T. Yepez-Martinez and A. P. Szczepaniak, Charmonium meson and hybrid radiative transitions, Phys. Rev. D 89, 116005 (2014).

[41] P. Gonzalez, Charmonium description from a generalized screened potential model, Phys. Rev. D 92, 014017 (2015).

[42] G. Li and Q. Zhao, Hadronic loop contributions to $J / \psi$ and $\psi^{\prime}$ radiative decays into $\gamma \eta_{c}$ or $\gamma \eta_{c}^{\prime}$, Phys. Lett. B 670, 55 (2008).

[43] G. Li and Q. Zhao, Revisit the radiative decays of $J / \psi$ and $\psi^{\prime} \rightarrow \gamma \eta_{c}\left(\gamma \eta_{c}^{\prime}\right)$, Phys. Rev. D 84, 074005 (2011).

[44] C. W. Zhao, G. Li, X. H. Liu and F. L. Shao, Effects of heavy meson loops on heavy quarkonium radiative transitions, Eur. Phys. J. C 73, 2482 (2013).

[45] M. Ablikim et al. [BESIII Collaboration], Measurement of the branching fraction for $\psi(3770) \rightarrow \gamma \chi_{c 0}$, Phys. Lett. B 753, 103 (2016).

[46] E. Laermann, F. Langhammer, I. Schmitt and P. M. Zerwas, The Interquark Potential: SU(2) Color Gauge Theory With Fermions, Phys. Lett. B 173, 437 (1986).

[47] K. D. Born, E. Laermann, N. Pirch, T. F. Walsh and P. M. Zerwas, Hadron Properties in Lattice QCD With Dynamical Fermions, Phys. Rev. D 40, 1653 (1989).

[48] B. Q. Li and K. T. Chao, Bottomonium Spectrum with Screened Potential, Commun. Theor. Phys. 52, 653 (2009).

[49] Chong-Hai Cai and Lei Li, Radial equation of bound state and binding energies of $\Xi^{-}$hypernuclei, High Energy Physics and Nuclear Physics 27, 1005 (2003).

[50] W. J. Deng, H. Liu, L. C. Gui and X. H. Zhong, Spectrum and electromagnetic transitions of bottomonium,
arXiv:1607.04696 [hep-ph].

[51] M. Ablikim et al., Precise measurement of the $e^{+} e^{-} \rightarrow$ $\pi^{+} \pi^{-} J / \psi$ cross section at center-of-mass energies from 3.77 to $4.60 \mathrm{GeV}$, arXiv:1611.01317 [hep-ex].

[52] R. Aaij et al. [LHCb Collaboration], Observation of $J / \psi \phi$ structures consistent with exotic states from amplitude analysis of $B^{+} \rightarrow J / \psi \phi K^{+}$decays, arXiv:1606.07895 [hep-ex].

[53] F. K. Guo and U. G. Meissner, Where is the $\chi_{c 0}(2 P)$ ?, Phys. Rev. D 86, 091501 (2012).

[54] S. L. Olsen, Is the $X(3915)$ the $\chi_{c 0}(2 P)$ ?, Phys. Rev. D 91, 057501 (2015).

[55] Z. Y. Zhou, Z. Xiao and H. Q. Zhou, Could the X(3915) and the $X(3930)$ Be the Same Tensor State?, Phys. Rev. Lett. 115, 022001 (2015).

[56] S. J. Brodsky and J. R. Primack, The Electromagnetic Interactions of Composite Systems, Annals Phys. 52, 315 (1969).

[57] F. E. Close and L. A. Copley, Nucl. Phys. B 19, 477 (1970). doi:10.1016/0550-3213(70)90362-7

[58] Z. P. Li, The Threshold pion photoproduction of nucleons in the chiral quark model, Phys. Rev. D 50, 5639 (1994).

[59] Z. P. Li, H. X. Ye and M. H. Lu, An unified approach to pseudoscalar meson photoproductions off nucleons in the quark model, Phys. Rev. C 56, 1099 (1997).

[60] Z. P. Li, The Kaon photoproduction of nucleons in the chiral quark model, Phys. Rev. C 52, 1648 (1995).

[61] Q. Zhao, Eta-prime photoproduction near threshold, Phys. Rev. C 63, 035205 (2001).

[62] B. Saghai and Z. p. Li, Quark model study of the eta photoproduction: Evidence for a new $S 11$ resonance?, Eur. Phys. J. 
A 11, 217 (2001).

[63] Q. Zhao, J. S. Al-Khalili, Z. P. Li and R. L. Workman, Pion photoproduction on the nucleon in the quark model, Phys. Rev. C 65, 065204 (2002).

[64] J. He, B. Saghai and Z. Li, Study of $\eta$ photoproduction on the proton in a chiral constituent quark approach via one-gluonexchange model, Phys. Rev. C 78, 035204 (2008).

[65] J. He and B. Saghai, Combined study of $\gamma p \rightarrow \eta p$ and $\pi^{-} p \rightarrow$ $\eta n$ in a chiral constituent quark approach, Phys. Rev. C 80, 015207 (2009).

[66] J. He and B. Saghai, $\eta$ production off the proton in a Reggeplus-chiral quark approach, Phys. Rev. C 82, 035206 (2010).

[67] X. H. Zhong and Q. Zhao, $\eta$ photoproduction on the quasi-free nucleons in the chiral quark model, Phys. Rev. C 84, 045207 (2011).

[68] X. H. Zhong and Q. Zhao, $\eta^{\prime}$ photoproduction on the nucleons in the quark model, Phys. Rev. C 84, 065204 (2011).

[69] L. Y. Xiao, X. Cao and X. H. Zhong, Neutral pion photoproduction on the nucleon in a chiral quark model, Phys. Rev. C 92, no. 3, 035202 (2015).

[70] Q. Zhao, Z. p. Li and C. Bennhold, Vector meson photoproduction with an effective Lagrangian in the quark model, Phys. Rev. C 58, 2393 (1998).

[71] Q. Zhao, Z. p. Li and C. Bennhold, Omega and rho photoproduction with an effective quark model Lagrangian, Phys. Lett. B 436, 42 (1998).

[72] W. J. Deng, L. Y. Xiao, L. C. Gui and X. H. Zhong, Radiative transitions of charmonium states in a constituent quark model, arXiv:1510.08269 [hep-ph].

[73] L. Durand, P. C. DeCelles and R. B. Marr, Lorentz Invariance and the Kinematic Structure of Vertex Functions, Phys. Rev. 126, 1882 (1962).

[74] G. Karl, S. Meshkov and J. L. Rosner, Symmetries, Angular Distributions in $\psi^{\prime} \rightarrow \gamma \chi \rightarrow \gamma \gamma \psi$, and the Interpretation of the $\chi(3400$ - 3550) Levels, Phys. Rev. D 13, 1203 (1976).

[75] M. G. Olsson and C. J. Suchyta, III, Radiative Angular Distributions From $c \bar{c}$ States Directly Produced by $p \bar{p}$ Annihilation, Phys. Rev. D 34, 2043 (1986).

[76] F. E. Close, A. Donnachie and Y. S. Kalashnikova, Radiative decays: A New flavor filter, Phys. Rev. D 67, 074031 (2003).

[77] F. E. Close, A. Donnachie and Y. S. Kalashnikova, Radiative decays of excited vector mesons, Phys. Rev. D 65, 092003 (2002).

[78] R. Bonnaz, B. Silvestre-Brac and C. Gignoux, Radiative transitions in mesons in a nonrelativistic quark model, Eur. Phys. J. A 13, 363 (2002).

[79] V. V. Anashin et al., Measurement of $J / \psi \rightarrow \gamma \eta_{\mathrm{c}}$ decay rate and $\eta_{\mathrm{c}}$ parameters at KEDR, Phys. Lett. B 738, 391 (2014).

[80] M. Ablikim et al. [BESIII Collaboration], Measurement of $\mathcal{B}\left(\psi(3770) \rightarrow \gamma \chi_{c 1}\right)$ and search for $\psi(3770) \rightarrow \gamma \chi_{c 2}$, Phys. Rev. D 91, 092009 (2015).

[81] V. Bhardwaj et al. [Belle Collaboration], Evidence of a new narrow resonance decaying to $\chi_{c 1} \gamma$ in $B \rightarrow \chi_{c 1} \gamma K$, Phys. Rev. Lett. 111, 032001 (2013).

[82] M. Ablikim et al. [BESIII Collaboration], Observation of the $\psi\left(1^{3} D_{2}\right)$ state in $e^{+} e^{-} \rightarrow \pi^{+} \pi^{-} \gamma \chi_{c 1}$ at BESIII, Phys. Rev. Lett. 115, 011803 (2015).

[83] C. F. Qiao, F. Yuan and K. T. Chao, A Crucial test for color octet production mechanism in $Z^{0}$ decays, Phys. Rev. D 55, 4001 (1997).

[84] H. Xu, X. Liu and T. Matsuki, Understanding $B^{-} \rightarrow$ $X(3823) K^{-}$via rescattering mechanism and predicting $B^{-} \rightarrow$ $\eta_{c 2}\left({ }^{1} D_{2}\right) / \psi_{3}\left({ }^{3} D_{3}\right) K^{-}$, Phys. Rev. D 94, 034005 (2016).

[85] Y. Fan, Z. G. He, Y. Q. Ma and K. T. Chao, Predictions of
Light Hadronic Decays of Heavy Quarkonium 1D(2) States in NRQCD, Phys. Rev. D 80, 014001 (2009).

[86] S. J. Sang, J. Z. Li, C. Meng and K. T. Chao, B meson semi-inclusive decay to spin-triplet $\mathrm{D}$-wave charmonium in NRQCD, Phys. Rev. D 91, 114023 (2015).

[87] S. Uehara et al. [Belle Collaboration], Observation of a $\chi_{c 2}^{\prime}$ candidate in $\gamma \gamma \rightarrow D \bar{D}$ production at BELLE, Phys. Rev. Lett. 96, 082003 (2006).

[88] B. Aubert et al. [BaBar Collaboration], Observation of the $\chi_{c 2}(2 p)$ Meson in the Reaction $\gamma \gamma \rightarrow D \bar{D}$ at BaBar, Phys. Rev. D 81, 092003 (2010).

[89] K. T. Chao, Z. G. He, D. Li and C. Meng, Search for $C=$ + charmonium states in $e^{+} e^{-} \rightarrow \gamma+X$ at BEPCII/BESIII, arXiv:1310.8597 [hep-ph].

[90] S. L. Olsen, A New Hadron Spectroscopy, Front. Phys. China. 10, 121 (2015).

[91] B. Aubert et al. [BaBar Collaboration], Evidence for $X(3872) \rightarrow \psi(2 S) \gamma$ in $B^{ \pm} \rightarrow X(3872) K^{ \pm}$decays, and a study of $B \rightarrow c \bar{c} \gamma K$, Phys. Rev. Lett. 102, 132001 (2009).

[92] V. Bhardwaj et al. [Belle Collaboration], Observation of $X(3872) \rightarrow J / \psi \gamma$ and search for $X(3872) \rightarrow \psi^{\prime} \gamma$ in B decays, Phys. Rev. Lett. 107, 091803 (2011).

[93] R. Aaij et al. [LHCb Collaboration], Evidence for the decay $X(3872) \rightarrow \psi(2 S) \gamma$, Nucl. Phys. B 886, 665 (2014).

[94] C. Meng, Y. J. Gao and K. T. Chao, B ${ }_{c 1}(1 \mathrm{P}, 2 \mathrm{P}) \mathrm{K}$ decays in QCD factorization and X(3872), Phys. Rev. D 87, 074035 (2013).

[95] X. Liu, Z. G. Luo and Z. F. Sun, $X(3915)$ and $X(4350)$ as new members in P-wave charmonium family, Phys. Rev. Lett. 104, 122001 (2010).

[96] Y. L. Han et al. [Belle Collaboration], Measurement of $e^{+} e^{-} \rightarrow \gamma \chi_{c J}$ via initial state radiation at Belle, Phys. Rev. D 92, 012011 (2015).

[97] [BESIII Collaboration], Observation of two resonant structures in $e^{+} e^{-} \rightarrow \pi^{+} \pi^{-} h_{c}$, arXiv:1610.07044 [hep-ex].

[98] M. Ablikim et al. [BESIII Collaboration], Study of $e^{+} e^{-} \rightarrow$ $\omega \chi_{c J}$ at center-of-mass energies from 4.21 to $4.42 \mathrm{GeV}$, Phys. Rev. Lett. 114, 092003 (2015).

[99] The 4th workshop on the XYZ particles, November 22-25, 2016, Beihang University, http://indico.ihep.ac.cn/event/6236/

[100] J. Z. Bai et al. [BES Collaboration], Measurements of the cross-section for $e^{+} e^{-} \rightarrow$ hadrons at center-of-mass energies from $2 \mathrm{GeV}$ to $5 \mathrm{GeV}$, Phys. Rev. Lett. 88, 101802 (2002).

[101] X. H. Mo, C. Z. Yuan and P. Wang, On the leptonic partial widths of the excited $\psi$ states, Phys. Rev. D 82, 077501 (2010).

[102] D. Cronin-Hennessy et al. [CLEO Collaboration], Measurement of Charm Production Cross Sections in $e^{+} e^{-}$Annihilation at Energies between 3.97 and $4.26 \mathrm{GeV}$, Phys. Rev. D 80, 072001 (2009).

[103] G. Karl, S. Meshkov and J. L. Rosner, Quark Magnetic Moments and E1 Radiative Transitions in Charmonium, Phys. Rev. Lett. 45, 215 (1980).

[104] H. Grotch, D. A. Owen and K. J. Sebastian, Relativistic Corrections to Radiative Transitions and Spectra of Quarkonia, Phys. Rev. D 30, 1924 (1984).

[105] S. Godfrey, G. Karl and P. J. O’Donnell, S Wave Triplet $D$ Wave Triplet Mixing and E1 Radiative Transitions in Charmonium, Z. Phys. C 31, 77 (1986).

[106] M. A. Doncheski, H. Grotch and K. J. Sebastian, Angular distribution of photons in the parity changing one photon radiative decays of quarkonia, Phys. Rev. D 42, 2293 (1990).

[107] K. J. Sebastian, H. Grotch and F. L. Ridener, Multipole amplitudes in parity changing one photon transitions of charmo- 
nium, Phys. Rev. D 45, 3163 (1992).

[108] R. McClary and N. Byers, Relativistic Effects in Heavy Quarkonium Spectroscopy, Phys. Rev. D 28, 1692 (1983).

[109] J. L. Rosner, M2 signatures in $\psi(2 S)$ radiative decays, Phys. Rev. D 78, 114011 (2008).

[110] M. Oreglia et al., A Study of the Reaction $\psi^{\prime} \rightarrow \gamma \gamma J / \psi$, Phys. Rev. D 25, 2259 (1982).

[111] T. A. Armstrong et al. [E760 Collaboration], Study of the angular distribution of the reaction $\bar{p} p \rightarrow \chi_{c 2} \rightarrow J / \psi \gamma \rightarrow e^{+} e^{-} \gamma$, Phys. Rev. D 48, 3037 (1993).

[112] M. Ambrogiani et al. [E835 Collaboration], Study of the angular distributions of the reactions $\bar{p} p \rightarrow \chi_{c 1}, \chi_{c 2} \rightarrow J / \psi \gamma \rightarrow$ $e^{+} e^{-} \gamma$, Phys. Rev. D 65, 052002 (2002).

[113] M. Ablikim et al. [BES Collaboration], Measurement of the chi(c2) polarization in $\psi(2 S) \rightarrow \gamma \chi_{c 2}$, Phys. Rev. D 70, 092004 (2004).

[114] M. Artuso et al. [CLEO Collaboration], Higher-order multipole amplitudes in charmonium radiative transitions, Phys. Rev. D 80, 112003 (2009).

[115] M. Ablikim et al. [BESIII Collaboration], Higher-order multipole amplitude measurement in $\psi(2 S) \rightarrow \gamma \chi_{c 2}$, Phys. Rev. D 84, 092006 (2011). 
TABLE VIII: Partial widths $\Gamma(\mathrm{keV})$ and branching ratios $B r$ for the radiative transitions (E1 dominant) of the higher $2 P$ and $3 P$ states. LP and SP stand for our results obtained from the linear potential and screened potential models, respectively. For comparison, the predictions from the NR and GI models [8] and SNR model [11] are listed in the table as well.

\begin{tabular}{|c|c|c|c|c|c|c|c|c|c|c|c|c|}
\hline \multirow{2}{*}{$\begin{array}{l}\text { Initial } \\
\text { state }\end{array}$} & \multirow{2}{*}{$\begin{array}{l}\text { Final } \\
\text { state }\end{array}$} & \multirow{2}{*}{\multicolumn{2}{|c|}{ 8] $\frac{E_{\gamma}(\mathrm{MeV})}{\mathrm{SNR}[11]}$}} & \multirow[b]{2}{*}{$\mathrm{LP} / \mathrm{SP}$} & \multicolumn{4}{|c|}{$\Gamma_{\mathrm{E} 1}(\mathrm{keV})$} & \multicolumn{2}{|c|}{$\Gamma_{\mathrm{EM}}(\mathrm{keV})$} & \multicolumn{2}{|c|}{$\underline{B r}$} \\
\hline & & & & & NR/GI [8] & $\mathrm{SNR}_{0 / 1}[11]$ & LP & SP & LP & SP & LP & SP \\
\hline$\chi_{c 2}(2 P)$ & $\psi_{3}(1 D)$ & $163 / 128$ & & $96 / 96$ & $88 / 29$ & & 20 & 24 & 20 & 24 & $8.3 \times 10^{-4}$ & $1.0 \times 10^{-3}$ \\
\hline \multirow[t]{4}{*}{$24 \pm 6^{a}$} & $\psi_{2}(1 D)$ & $168 / 139$ & & 103 & $17 / 5.6$ & & 3.3 & 4.1 & 3.2 & 4.0 & $1.3 \times 10^{-4}$ & $1.7 \times 10^{-4}$ \\
\hline & $\psi_{1}(1 D)$ & 197 /204 & & 146 & $1.9 / 1.0$ & & 0.47 & 0.62 & 0.36 & 0.46 & $1.5 \times 10^{-5}$ & $1.9 \times 10^{-5}$ \\
\hline & $\psi(2 S)$ & $276 / 282$ & 235 & 234 & 304 / 207 & $225 / 100$ & 146 & 163 & 135 & 150 & $5.6 \times 10^{-3}$ & $6.3 \times 10^{-3}$ \\
\hline & $J / \psi$ & 779 /784 & 744 & 742 & $81 / 53$ & $101 / 109$ & 118 & 119 & 93 & 93 & $3.9 \times 10^{-3} 3$ & $3.9 \times 10^{-3}$ \\
\hline$\chi_{c 1}(2 P)$ & $\psi_{2}(1 D)$ & $123 / 113$ & & $76 / 76$ & $35 / 18$ & & 2.8 & 3.4 & 2.9 & 3.5 & $1.8 \times 10^{-5}$ & $2.1 \times 10^{-5}$ \\
\hline \multirow[t]{3}{*}{$165^{b}$} & $\psi_{1}(1 D)$ & $152 / 179$ & & $120 / 120$ & $22 / 21$ & & 8.6 & 10.8 & 7.9 & 9.8 & $4.9 \times 10^{-5}$ & $5.9 \times 10^{-5}$ \\
\hline & $\psi(2 S)$ & $232 / 258$ & 182 & $208 / 208$ & 183 / 183 & $103 / 60$ & 129 & 145 & 139 & 155 & $8.4 \times 10^{-4}$ & $9.4 \times 10^{-4}$ \\
\hline & $J / \psi$ & $741 / 763$ & 697 & $720 / 720$ & $71 / 14$ & $83 / 45$ & 64 & 68 & 81 & 88 & $4.9 \times 10^{-4}$ & $5.3 \times 10^{-4}$ \\
\hline$\chi_{c 0}(2 P)$ & $\psi_{1}(1 D)$ & $81 / 143$ & & $90 / 69$ & 13 / 51 & & 21 & 12 & 20 & 12 & $6.7 \times 10^{-4}$ & $4.0 \times 10^{-4}$ \\
\hline \multirow[t]{2}{*}{$30^{b}$} & $\psi(2 S)$ & $162 / 223$ & 152 & $179 / 159$ & 64 / 135 & $61 / 44$ & 108 & 89 & 121 & 99 & $4.0 \times 10^{-3}$ & $3.3 \times 10^{-3}$ \\
\hline & $J / \psi$ & $681 / 733$ & 672 & $695 / 678$ & $56 / 1.3$ & $74 / 9.3$ & 4.0 & 1.5 & 6.1 & 2.3 & $2.0 \times 10^{-4}$ & $7.7 \times 10^{-5}$ \\
\hline \multirow{3}{*}{$\begin{array}{c}h_{c}(2 P) \\
87^{b}\end{array}$} & $\eta_{c 2}(1 D)$ & $133 / 117$ & & $100 / 100$ & $60 / 27$ & & 25 & 25 & 25 & 25 & $2.9 \times 10^{-4}$ & $2.9 \times 10^{-4}$ \\
\hline & $\eta_{c}(2 S)$ & $285 / 305$ & 261 & $252 / 252$ & 280 / 218 & $309 / 108$ & 160 & 176 & 160 & 176 & $1.8 \times 10^{-3}$ & $2.0 \times 10^{-3}$ \\
\hline & $\eta_{c}(1 S)$ & 839 /856 & 818 & $808 / 808$ & $140 / 85$ & $134 / 250$ & 135 & 134 & 135 & 134 & $1.6 \times 10^{-3}$ & $1.6 \times 10^{-3}$ \\
\hline \multirow{9}{*}{$\begin{array}{c}\chi_{c 2}(3 P) \\
66^{b}\end{array}$} & $\psi_{3}(2 D)$ & $147 / 118$ & & 136/98 & $148 / 51$ & & 116 & 64 & 121 & 66 & $1.8 \times 10^{-3}$ & $1.0 \times 10^{-3}$ \\
\hline & $\psi_{2}(2 D)$ & $156 / 127$ & & $143 / 101$ & $31 / 10$ & & 18 & 10 & 18 & 10 & $2.7 \times 10^{-4}$ & $1.5 \times 10^{-4}$ \\
\hline & $\psi_{1}(2 D)$ & $155 / 141$ & & $117 / 20$ & $2.1 / 0.77$ & & 0.55 & 0.004 & 0.44 & 0.004 & $6.7 \times 10^{-6}$ & $6.0 \times 10^{-8}$ \\
\hline & $\psi_{3}(1 D)$ & $481 / 461$ & & $453 / 364$ & 0.049 / 6.8 & & 15 & 10 & 17 & 12 & $1.1 \times 10^{-4}$ & $1.8 \times 10^{-4}$ \\
\hline & $\psi_{2}(1 D)$ & $486 / 470$ & & $459 / 370$ & $0.01 / 0.13$ & & 4.6 & 2.5 & 4.6 & 2.4 & $7.0 \times 10^{-5} 3$ & $3.6 \times 10^{-5}$ \\
\hline & $\psi_{1}(1 D)$ & $512 / 530$ & & $495 / 411$ & $0.00 / 0.00$ & & 1.9 & 1.0 & 1.5 & 0.79 & $2.2 \times 10^{-5}$ & $1.2 \times 10^{-5}$ \\
\hline & $\psi(3 S)$ & $268 / 231$ & & $261 / 168$ & 509 / 199 & & 306 & 121 & 281 & 114 & $4.3 \times 10^{-3}$ & $1.7 \times 10^{-3}$ \\
\hline & $\psi(2 S)$ & $585 / 602$ & & $574 / 492$ & $55 / 30$ & & 116 & 90 & 97 & 76 & $1.5 \times 10^{-3}$ & $1.2 \times 10^{-3}$ \\
\hline & $J / \psi$ & $1048 / 1063$ & & $1042 / 967$ & $34 / 19$ & & 83 & 69 & 61 & 51 & $9.2 \times 10^{-4}$ & $7.7 \times 10^{-4}$ \\
\hline \multirow{7}{*}{$\begin{array}{c}\chi_{c 1}(3 P) \\
39^{b}\end{array}$} & $\psi_{2}(2 D)$ & $112 / 108$ & & $117 / 82$ & $58 / 35$ & & 22 & 11 & 23 & 11 & $5.9 \times 10^{-4}$ & $2.8 \times 10^{-4}$ \\
\hline & $\psi_{1}(2 D)$ & $111 / 121$ & & $92 / 1$ & $19 / 15$ & & 8.6 & 0 & 8.1 & 0 & $2.1 \times 10^{-4}$ & 0 \\
\hline & $\psi_{2}(1 D)$ & $445 / 452$ & & $436 / 353$ & $0.035 / 4.6$ & & 0.13 & 0.09 & 0.12 & 0.09 & $3.1 \times 10^{-6}$ & $2.3 \times 10^{-6}$ \\
\hline & $\psi_{1}(1 D)$ & $472 / 512$ & & $476 / 394$ & $0.014 / 0.39$ & & 4.4 & 2.7 & 3.2 & 2.0 & $6.1 \times 10^{-5}$ & $4.1 \times 10^{-5}$ \\
\hline & $\psi(3 S)$ & $225 / 212$ & & $237 / 149$ & 303 / 181 & & 305 & 111 & 331 & 117 & $8.5 \times 10^{-3} 3$ & $3.0 \times 10^{-3}$ \\
\hline & $\psi(2 S)$ & $545 / 585$ & & $556 / 475$ & 45 / 8.9 & & 78 & 63 & 94 & 74 & $2.4 \times 10^{-3}$ & $1.9 \times 10^{-3}$ \\
\hline & $J / \psi$ & $1013 / 1048$ & & $1023 / 952$ & $31 / 2.2$ & & 36 & 33 & 50 & 45 & $1.3 \times 10^{-3}$ & $1.2 \times 10^{-3}$ \\
\hline \multirow{5}{*}{$\begin{array}{c}\chi_{c 0}(3 P) \\
51^{b}\end{array}$} & $\psi_{1}(2 D)$ & $43 / 97$ & & $39 / 45$ & 4.4 / 35 & & 3.8 & 9.3 & 3.8 & 9.1 & $7.5 \times 10^{-5}$ & $1.8 \times 10^{-4}$ \\
\hline & $\psi_{1}(1 D)$ & $410 / 490$ & & $427 / 352$ & 0.037 / 9.7 & & 0.31 & 0.44 & 0.27 & 0.39 & $5.3 \times 10^{-6}$ & $7.6 \times 10^{-6}$ \\
\hline & $\psi(3 S)$ & $159 / 188$ & & $186 / 105$ & 109 / 145 & & 214 & 56 & 241 & 61 & $4.7 \times 10^{-3}$ & $1.2 \times 10^{-3}$ \\
\hline & $\psi(2 S)$ & $484 / 563$ & & $509 / 434$ & $32 / 0.045$ & & 13 & 6.9 & 17 & 9.1 & $3.3 \times 10^{-4}$ & $1.8 \times 10^{-4}$ \\
\hline & $J / \psi$ & $960 / 1029$ & & $981 / 916$ & $27 / 1.5$ & & 0.14 & 0.08 & 0.24 & 0.13 & $4.7 \times 10^{-6}$ & $2.5 \times 10^{-6}$ \\
\hline \multirow{5}{*}{$\begin{array}{c}h_{c}(3 P) \\
75^{b}\end{array}$} & $\eta_{c 2}(2 D)$ & $119 / 109$ & & $120 / 84$ & $99 / 48$ & & 93 & 47 & 93 & 47 & $1.2 \times 10^{-3}$ & $6.3 \times 10^{-4}$ \\
\hline & $\eta_{c 2}(1 D)$ & $453 / 454$ & & $453 / 370$ & $0.16 / 5.7$ & & 15 & 8.7 & 15 & 8.7 & $2.0 \times 10^{-4}$ & $1.2 \times 10^{-4}$ \\
\hline & $\eta_{c}(3 S)$ & $229 / 246$ & & $238 / 185$ & 276 / 208 & & 237 & 146 & 237 & 146 & $3.2 \times 10^{-3}$ & $1.9 \times 10^{-3}$ \\
\hline & $\eta_{c}(2 S)$ & $593 / 627$ & & $602 / 517$ & 75 / 43 & & 124 & 96 & 124 & 96 & $1.7 \times 10^{-3}$ & $1.3 \times 10^{-3}$ \\
\hline & $\eta_{c}(1 S)$ & $1103 / 1131$ & & $1104 / 1035$ & $72 / 38$ & & 90 & 77 & 90 & 77 & $1.2 \times 10^{-4}$ & $1.0 \times 10^{-3}$ \\
\hline
\end{tabular}

${ }^{a}$ Width (MeV) from the PDG [6].

${ }^{b}$ Predicted width (MeV) from Ref. [8]. 
TABLE IX: Partial widths $\Gamma(\mathrm{keV})$ and branching ratios $B r$ for the radiative transitions (E1 dominant) of the higher $D$-wave states. LP and SP stand for our results obtained from the linear potential and screened potential models, respectively. For comparison, the predictions from the relativistic quark model [37], NR and GI models [8], and SNR model [11] are listed in the table as well.

\begin{tabular}{|c|c|c|c|c|c|c|c|c|c|c|c|c|c|c|}
\hline \multirow{2}{*}{$\begin{array}{c}\text { Initial } \\
\text { state }\end{array}$} & \multirow{2}{*}{$\begin{array}{l}\text { Final } \\
\text { state }\end{array}$} & \multicolumn{4}{|c|}{$\underline{E_{\gamma}(\mathrm{MeV})}$} & \multicolumn{5}{|c|}{$\underline{\Gamma_{\mathrm{E} 1}(\mathrm{keV})}$} & \multicolumn{2}{|c|}{$\Gamma_{\mathrm{EM}}(\mathrm{keV})$} & \multicolumn{2}{|c|}{$\underline{B r}$} \\
\hline & & [37] & NR/GI [8] & SNR [11] & $\mathrm{LP} / \mathrm{SP}$ & [37] & NR/GI [8] & $\mathrm{SNR}_{0 / 1}[11]$ & LP & $\mathrm{SP}$ & LP & SP & LP & SP \\
\hline$\psi_{3}(1 D)$ & $\chi_{c 2}(1 P)$ & 250 & $242 / 282$ & 236 & $264 / 264$ & 156 & $272 / 296$ & $284 / 223$ & 377 & 393 & 350 & 364 & $12 \%$ & $12 \%$ \\
\hline$\eta_{c 2}(1 D)$ & $h_{c}(1 P)$ & 275 & $264 / 307$ & 260 & $284 / 284$ & 245 & $339 / 344$ & $575 / 375$ & 362 & 376 & 362 & 376 & $72 \%$ & $75 \%$ \\
\hline$\psi_{3}(2 D)$ & $\chi_{c 2}(1 P)$ & & $566 / 609$ & & $571 / 518$ & & $29 / 16$ & & 83 & 78 & 72 & 67 & $4.9 \times 10^{-4}$ & $4.5 \times 10^{-4}$ \\
\hline $148^{a}$ & $\chi_{c 2}(2 P)$ & & $190 / 231$ & & $238 / 181$ & & $239 / 272$ & & 457 & 256 & 427 & 243 & $2.9 \times 10^{-3}$ & $1.6 \times 10^{-3}$ \\
\hline$\psi_{2}(2 D)$ & $\chi_{c 2}(1 P)$ & & $558 / 602$ & & $564 / 516$ & & $7.1 / 0.62$ & & 16 & 16 & 20 & 20 & $1.7 \times 10^{-4}$ & $2.2 \times 10^{-4}$ \\
\hline \multirow[t]{3}{*}{$92^{a}$} & $\chi_{c 1}(1 P)$ & & $597 / 640$ & & $603 / 554$ & & $26 / 23$ & & 64 & 64 & 68 & 68 & $7.4 \times 10^{-4}$ & $7.4 \times 10^{-4}$ \\
\hline & $\chi_{c 2}(2 P)$ & & $182 / 223$ & & $231 / 178$ & & $52 / 65$ & & 101 & 57 & 115 & 64 & $1.3 \times 10^{-3}$ & $7.0 \times 10^{-4}$ \\
\hline & $\chi_{c 1}(2 P)$ & & $226 / 247$ & & $222 / 204$ & & $298 / 225$ & & 220 & 186 & 223 & 188 & $2.4 \times 10^{-3}$ & $2.0 \times 10^{-3}$ \\
\hline$\psi_{1}(2 D)$ & $\chi_{c 2}(1 P)$ & & $559 / 590$ & & 587 & & $0.79 / 0.027$ & & 16 & 16 & 17 & 20 & $2.3 \times 10^{-4}$ & $2.7 \times 10^{-4}$ \\
\hline \multirow[t]{5}{*}{$74^{a}$} & $\chi_{c 1}(1 P)$ & & $598 / 628$ & & 625 & & $14 / 3.4$ & & 25 & 42 & 37 & 63 & $5.0 \times 10^{-4}$ & $8.5 \times 10^{-4}$ \\
\hline & $\chi_{c 0}(1 P)$ & & $677 / 707$ & & 704 & & $27 / 35$ & & 120 & 149 & 150 & 189 & $2.0 \times 10^{-3}$ & $2.6 \times 10^{-3}$ \\
\hline & $\chi_{c 2}(2 P)$ & & $183 / 210$ & & 256 & & $5.9 / 6.3$ & & 18 & 21 & 24 & 29 & $3.2 \times 10^{-4}$ & $3.9 \times 10^{-4}$ \\
\hline & $\chi_{c 1}(2 P)$ & & $227 / 234$ & & $281 / 281$ & & $168 / 114$ & & 253 & 280 & 309 & 347 & $4.2 \times 10^{-3}$ & $4.7 \times 10^{-3}$ \\
\hline & $\chi_{c 0}(2 P)$ & & $296 / 269$ & & $312 / 329$ & & $483 / 191$ & & 299 & 321 & 332 & 360 & $4.5 \times 10^{-3}$ & $4.9 \times 10^{-3}$ \\
\hline$\eta_{c 2}(2 D)$ & $h_{c}(1 P)$ & & $585 / 634$ & & $590 / 542$ & & $40 / 25$ & & 96 & 92 & 96 & 92 & $1.3 \times 10^{-3}$ & $1.2 \times 10^{-3}$ \\
\hline $111^{a}$ & $h_{c}(2 P)$ & & $218 / 244$ & & $256 / 203$ & & $336 / 296$ & & 438 & 271 & 438 & 271 & $3.9 \times 10^{-3}$ & $2.4 \times 10^{-3}$ \\
\hline
\end{tabular}

${ }^{a}$ Predicted width (MeV) from Ref. [8].

TABLE X: Three-photon decay chains of $2^{3} P_{2}$. The branching fractions are $B r_{1}=B r\left[2^{3} P_{2} \rightarrow 1^{3} D_{J} \gamma\right], B r_{2}=B r\left[1^{3} D_{J} \rightarrow 1^{3} P_{J} \gamma\right]$, $B r_{3}=B r\left[1^{3} P_{J} \rightarrow J / \psi \gamma\right]$, and $B r=B r_{1} \times B r_{2} \times B r_{3}$ is the combined branching fractions of the chain. The branching fractions are predicted with the linear potential model.

\begin{tabular}{c|c|c|c|c}
\hline \hline Decay chain & $B r_{1}$ & $B r_{2}$ & $B r_{3}$ & $B r$ \\
\hline $2^{3} P_{2} \rightarrow 1^{3} D_{1} \rightarrow 1^{3} P_{0} \rightarrow J / \psi$ & $1.5 \times 10^{-5}$ & $9.9 \times 10^{-3}$ & $1.6 \%$ & $2.4 \times 10^{-9}$ \\
$2^{3} P_{2} \rightarrow 1^{3} D_{1} \rightarrow 1^{3} P_{1} \rightarrow J / \psi$ & $1.5 \times 10^{-5}$ & $5.5 \times 10^{-3}$ & $34.8 \%$ & $2.9 \times 10^{-8}$ \\
$2^{3} P_{2} \rightarrow 1^{3} D_{1} \rightarrow 1^{3} P_{2} \rightarrow J / \psi$ & $1.5 \times 10^{-5}$ & $4.8 \times 10^{-4}$ & $14.6 \%$ & $1.0 \times 10^{-8}$ \\
$2^{3} P_{2} \rightarrow 1^{3} D_{2} \rightarrow 1^{3} P_{1} \rightarrow J / \psi$ & $1.3 \times 10^{-4}$ & $42 \%$ & $34.8 \%$ & $1.9 \times 10^{-5}$ \\
$2^{3} P_{2} \rightarrow 1^{3} D_{2} \rightarrow 1^{3} P_{2} \rightarrow J / \psi$ & $1.3 \times 10^{-4}$ & $13 \%$ & $14.6 \%$ & $2.5 \times 10^{-6}$ \\
$2^{3} P_{2} \rightarrow 1^{3} D_{3} \rightarrow 1^{3} P_{2} \rightarrow J / \psi$ & $8.3 \times 10^{-4}$ & $12 \%$ & $14.6 \%$ & $1.4 \times 10^{-5}$ \\
\hline \hline
\end{tabular}

TABLE XI: Two-photon decay chains of $3^{3} S_{1}$. The branching fractions are $B r_{1}=B r\left[3^{3} S_{1} \rightarrow 2^{3} P_{J} \gamma\right], B r_{2}=B r\left[2^{3} P_{J} \rightarrow 2^{3} S_{1} \gamma, J / \psi \gamma\right]$, $B r_{3}=\operatorname{Br}\left[2^{3} S_{1}, J / \psi \rightarrow \mu^{+} \mu^{-}\right]$(obtained from PDG [6]), and $B r=B r_{1} \times B r_{2} \times B r_{3}$ is the combined branching fractions of the chain. The theoretical branching fractions are predicted with the linear potential model. The estimated events are based on producing of $5 \times 10^{7} \psi(3 S)$ events at BESIII in coming years as described in the text.

\begin{tabular}{c|c|c|c|c|c}
\hline \hline Decay chain & $B r_{1}\left(10^{-4}\right)$ & $B r_{2}\left(10^{-4}\right)$ & $\operatorname{Br}(\%)$ & $\operatorname{Br}\left(10^{-8}\right)$ & Events \\
\hline $3^{3} S_{1} \rightarrow 2^{3} P_{2} \rightarrow 2^{3} S_{1} \rightarrow \mu^{+} \mu^{-}$ & 8.4 & 56 & 0.79 & 3.7 & 2 \\
$3^{3} S_{1} \rightarrow 2^{3} P_{1} \rightarrow 2^{3} S_{1} \rightarrow \mu^{+} \mu^{-}$ & 6.9 & 8.4 & 0.79 & 0.46 & 0.2 \\
$3^{3} S_{1} \rightarrow 2^{3} P_{0} \rightarrow 2^{3} S_{1} \rightarrow \mu^{+} \mu^{-}$ & 2.4 & 40 & 0.79 & 0.76 & 0.4 \\
$3^{3} S_{1} \rightarrow 2^{3} P_{2} \rightarrow J / \psi \rightarrow \mu^{+} \mu^{-}$ & 8.4 & 39 & 5.9 & 19 & 10 \\
$3^{3} S_{1} \rightarrow 2^{3} P_{1} \rightarrow J / \psi \rightarrow \mu^{+} \mu^{-}$ & 6.9 & 4.9 & 5.9 & 2.0 & 1 \\
$3^{3} S_{1} \rightarrow 2^{3} P_{0} \rightarrow J / \psi \rightarrow \mu^{+} \mu^{-}$ & 2.4 & 2.0 & 5.9 & 0.28 & 0.1 \\
\hline \hline
\end{tabular}


TABLE XII: Two-photon decay chains of $2 D$-wave states. The branching fractions are $B r_{1}=B r[2 D \rightarrow n P \gamma], B r_{2}=B r\left[n P \rightarrow 2^{3} S_{1} \gamma, 1 S \gamma\right]$, $B r_{3}=B r\left[2^{3} S_{1}, J / \psi \rightarrow \mu^{+} \mu^{-}\right]$(obtained from PDG [6]) or $B r_{3}=B r\left[\eta_{c}(1 S) \rightarrow K \bar{K} \pi\right]$ (obtained from PDG [6]), and $B r=B r_{1} \times B r_{2} \times B r_{3}$ is the combined branching fractions of the chain. The theoretical branching fractions are predicted with the linear potential model. The estimated events are based on producing of $2.4 \times 10^{7} \psi(4160)$ 's at BESIII as described in the text.

\begin{tabular}{|c|c|c|c|c|c|}
\hline Decay chain & $B r_{1}\left(10^{-3}\right)$ & $B r_{2}\left(10^{-4}\right)$ & $\mathrm{Br}_{3}(\%)[6]$ & $\operatorname{Br}\left(10^{-7}\right)$ & Events \\
\hline $2^{3} D_{1} \rightarrow 2^{3} P_{2} \rightarrow 2^{3} S_{1} \rightarrow \mu^{+} \mu^{-}$ & 0.32 & 56 & 0.79 & 0.14 & 0.3 \\
\hline $2^{3} D_{1} \rightarrow 2^{3} P_{1} \rightarrow 2^{3} S_{1} \rightarrow \mu^{+} \mu^{-}$ & 4.2 & 8.4 & 0.79 & 0.28 & 0.6 \\
\hline $2^{3} D_{1} \rightarrow 2^{3} P_{0} \rightarrow 2^{3} S_{1} \rightarrow \mu^{+} \mu^{-}$ & 4.5 & 40 & 0.79 & 1.4 & 3 \\
\hline $2^{3} D_{1} \rightarrow 2^{3} P_{2} \rightarrow J / \psi \rightarrow \mu^{+} \mu^{-}$ & 0.32 & 39 & 5.9 & 0.74 & 2 \\
\hline $2^{3} D_{1} \rightarrow 2^{3} P_{1} \rightarrow J / \psi \rightarrow \mu^{+} \mu^{-}$ & 4.2 & 4.9 & 5.9 & 1.2 & 3 \\
\hline $2^{3} D_{1} \rightarrow 2^{3} P_{0} \rightarrow J / \psi \rightarrow \mu^{+} \mu^{-}$ & 4.5 & 2.0 & 5.9 & 0.53 & 1 \\
\hline $2^{3} D_{1} \rightarrow 1^{3} P_{2} \rightarrow J / \psi \rightarrow \mu^{+} \mu^{-}$ & 0.23 & 1460 & 5.9 & 19.8 & 47 \\
\hline $2^{3} D_{1} \rightarrow 1^{3} P_{1} \rightarrow J / \psi \rightarrow \mu^{+} \mu^{-}$ & 0.50 & 3480 & 5.9 & 102 & 244 \\
\hline $2^{3} D_{1} \rightarrow 1^{3} P_{0} \rightarrow J / \psi \rightarrow \mu^{+} \mu^{-}$ & 2.0 & 160 & 5.9 & 18.8 & 45 \\
\hline $2^{3} D_{2} \rightarrow 2^{3} P_{2} \rightarrow 2^{3} S_{1} \rightarrow \mu^{+} \mu^{-}$ & 1.3 & 56 & 0.79 & 0.57 & $\cdots$ \\
\hline $2^{3} D_{2} \rightarrow 2^{3} P_{1} \rightarrow 2^{3} S_{1} \rightarrow \mu^{+} \mu^{-}$ & 2.4 & 8.4 & 0.79 & 0.16 & $\cdots$ \\
\hline $2^{3} D_{2} \rightarrow 2^{3} P_{2} \rightarrow J / \psi \rightarrow \mu^{+} \mu^{-}$ & 1.3 & 39 & 5.9 & 3.0 & $\cdots$ \\
\hline $2^{3} D_{2} \rightarrow 2^{3} P_{1} \rightarrow J / \psi \rightarrow \mu^{+} \mu^{-}$ & 2.4 & 4.9 & 5.9 & 6.9 & $\cdots$ \\
\hline $2^{3} D_{2} \rightarrow 1^{3} P_{2} \rightarrow J / \psi \rightarrow \mu^{+} \mu^{-}$ & 0.17 & 1460 & 5.9 & 14.6 & $\cdots$ \\
\hline $2^{3} D_{2} \rightarrow 1^{3} P_{1} \rightarrow J / \psi \rightarrow \mu^{+} \mu^{-}$ & 0.74 & 3480 & 5.9 & 152 & . \\
\hline $2^{3} D_{3} \rightarrow 2^{3} P_{2} \rightarrow 2^{3} S_{1} \rightarrow \mu^{+} \mu^{-}$ & 2.9 & 56 & 0.79 & 1.3 & $\cdots$ \\
\hline $2^{3} D_{3} \rightarrow 2^{3} P_{2} \rightarrow J / \psi \rightarrow \mu^{+} \mu^{-}$ & 2.9 & 39 & 5.9 & 6.7 & $\cdots$ \\
\hline $2^{3} D_{3} \rightarrow 1^{3} P_{2} \rightarrow J / \psi \rightarrow \mu^{+} \mu^{-}$ & 0.49 & 1460 & 5.9 & 42 & $\cdots$ \\
\hline $2^{1} D_{2} \rightarrow 2^{1} P_{1} \rightarrow \eta_{c}(1 S) \rightarrow K \bar{K} \pi$ & 3.9 & 16 & 7.3 & 4.6 & $\cdots$ \\
\hline $2^{1} D_{2} \rightarrow 1^{1} P_{1} \rightarrow \eta_{c}(1 S) \rightarrow K \bar{K} \pi$ & 1.3 & 5100 & 7.3 & 483 & $\cdots$ \\
\hline
\end{tabular}

TABLE XIII: Two-photon decay chains of $4^{3} S_{1}$. The branching fractions are $B r_{1}=B r\left[4^{3} S_{1} \rightarrow 2^{3} P_{J} \gamma, 3^{3} P_{J} \gamma\right], B r_{2}=B r\left[n^{3} P_{J} \rightarrow m^{3} S_{1} \gamma\right]$, $\mathrm{Br}_{3}=\mathrm{Br}\left[2^{3} S_{1}, J / \psi \rightarrow \mu^{+} \mu^{-}\right]$(obtained from PDG [6]), and $\mathrm{Br}=\mathrm{Br}_{1} \times B r_{2} \times B r_{3}$ is the combined branching fractions of the chain. The theoretical branching fractions are predicted with the linear potential model. The estimated events are based on producing of $2 \times 10^{7} \psi(4 S)$ 's at BESIII in coming years as described in the text.

\begin{tabular}{c|c|c|c|c|c}
\hline \hline Decay chain & $B r_{1}\left(10^{-4}\right)$ & $B r_{2}\left(10^{-4}\right)$ & $B r_{3}(\%)[6]$ & $\operatorname{Br}\left(10^{-9}\right)$ & Events \\
\hline $4^{3} S_{1} \rightarrow 2^{3} P_{2} \rightarrow 2^{3} S_{1} \rightarrow \mu^{+} \mu^{-}$ & 1.7 & 56 & 0.79 & 7.5 & 0.15 \\
$4^{3} S_{1} \rightarrow 2^{3} P_{1} \rightarrow 2^{3} S_{1} \rightarrow \mu^{+} \mu^{-}$ & 2.6 & 8.4 & 0.79 & 1.7 & 0.03 \\
$4^{3} S_{1} \rightarrow 2^{3} P_{0} \rightarrow 2^{3} S_{1} \rightarrow \mu^{+} \mu^{-}$ & 1.5 & 40 & 0.79 & 4.7 & 0.09 \\
$4^{3} S_{1} \rightarrow 2^{3} P_{2} \rightarrow J / \psi \rightarrow \mu^{+} \mu^{-}$ & 1.7 & 39 & 5.9 & 39 & 0.78 \\
$4^{3} S_{1} \rightarrow 2^{3} P_{1} \rightarrow J / \psi \rightarrow \mu^{+} \mu^{-}$ & 2.6 & 4.9 & 5.9 & 7.5 & 0.15 \\
$4^{3} S_{1} \rightarrow 2^{3} P_{0} \rightarrow J / \psi \rightarrow \mu^{+} \mu^{-}$ & 1.5 & 2.0 & 5.9 & 1.8 & 0.03 \\
\hline $4^{3} S_{1} \rightarrow 3^{3} P_{2} \rightarrow 2^{3} S_{1} \rightarrow \mu^{+} \mu^{-}$ & 11 & 15 & 0.79 & 13 & 0.26 \\
$4^{3} S_{1} \rightarrow 3^{3} P_{1} \rightarrow 2^{3} S_{1} \rightarrow \mu^{+} \mu^{-}$ & 9.1 & 24 & 0.79 & 17 & 0.34 \\
$4^{3} S_{1} \rightarrow 3^{3} P_{0} \rightarrow 2^{3} S_{1} \rightarrow \mu^{+} \mu^{-}$ & 4.6 & 33 & 0.79 & 12 & 0.24 \\
$4^{3} S_{1} \rightarrow 3^{3} P_{2} \rightarrow J / \psi \rightarrow \mu^{+} \mu^{-}$ & 1.7 & 9.2 & 5.9 & 9.2 & 0.18 \\
$4^{3} S_{1} \rightarrow 3^{3} P_{1} \rightarrow J / \psi \rightarrow \mu^{+} \mu^{-}$ & 2.6 & 13 & 5.9 & 20 & 0.40 \\
$4^{3} S_{1} \rightarrow 3^{3} P_{0} \rightarrow J / \psi \rightarrow \mu^{+} \mu^{-}$ & 1.5 & 0.047 & 5.9 & 0.041 & 0 \\
\hline \hline
\end{tabular}


TABLE XIV: The predicted ratios between the magnetic quadrupole amplitude $a_{2}$ and the electric-dipole amplitude $a_{1}$ compared with the data. The predicted ratios between the "extra" electric-dipole $E_{R}$ and electric-dipole $a_{1}$ are also listed. LP and SP stand for our results obtained from the linear potential and screened potential models, respectively.

\begin{tabular}{|c|c|c|c|c|c|c|c|c|c|}
\hline process & $\begin{array}{l}\frac{E_{R}}{a_{1}} \\
\text { LP }\end{array}$ & $\begin{array}{l}\frac{E_{R}}{a_{1}} \\
\text { SP }\end{array}$ & $\begin{array}{l}\frac{a_{2}}{a_{1}} \\
\text { SP }\end{array}$ & $\begin{array}{l}\frac{a_{2}}{a_{1}} \\
\text { LP }\end{array}$ & $\begin{array}{c}\frac{a_{2}}{a_{1}} \\
\text { Lat. [20] }\end{array}$ & $\begin{array}{c}\frac{a_{2}}{a_{1}} \\
\text { CLEO [114] }\end{array}$ & $\begin{array}{c}\frac{a_{2}}{a_{1}} \\
\text { BESII [113] }\end{array}$ & $\begin{array}{c}\frac{a_{2}}{a_{1}} \\
\text { Crystal Ball [110] }\end{array}$ & $\begin{array}{c}\frac{a_{2}}{a_{1}} \\
\text { BESIII [115] }\end{array}$ \\
\hline$\chi_{c 1}(1 P) \rightarrow J / \psi \gamma$ & +0.062 & +0.065 & -0.065 & -0.062 & $-0.09(7)$ & $-0.0626(87)$ & & $-0.002_{-0.020}^{+0.008}$ & \\
\hline$\chi_{c 2}(1 P) \rightarrow J / \psi \gamma$ & -0.078 & -0.082 & -0.110 & -0.105 & $-0.39(7)$ & $-0.093(19)$ & & $-0.333_{-0.292}^{+0.116}$ & \\
\hline$\psi(2 S) \rightarrow \chi_{c 1}(1 P) \gamma$ & -0.030 & +0.031 & -0.031 & -0.030 & & $0.0276(96)$ & & $0.077_{-0.045}^{+0.050}$ & \\
\hline$\psi(2 S) \rightarrow \chi_{c 2}(1 P) \gamma$ & +0.021 & +0.022 & -0.030 & -0.028 & & $0.010(16)$ & $-0.051_{-0.036}^{+0.054}$ & $0.132_{-0.075}^{+0.098}$ & $0.046(23)$ \\
\hline
\end{tabular}

TABLE XV: The predicted ratios $\frac{a_{2}}{a_{1}}$ and $\frac{E_{R}}{a_{1}}$ with the linear potential (LP) and screened potential (SP) models.

\begin{tabular}{c|llll}
\hline \hline process & $\frac{E_{R}}{a_{1}}(\mathrm{LP})$ & $\frac{E_{R}}{a_{1}}(\mathrm{SP})$ & $\frac{a_{2}}{a_{1}}(\mathrm{SP})$ & $\frac{a_{2}}{a_{1}}(\mathrm{LP})$ \\
\hline$\psi_{1}(1 D) \rightarrow \chi_{c 1}(1 P) \gamma$ & +0.088 & +0.092 & +0.041 & +0.040 \\
$\psi_{1}(1 D) \rightarrow \chi_{c 2}(1 P) \gamma$ & +0.214 & +0.224 & +0.074 & +0.066 \\
$\chi_{c 1}(2 P) \rightarrow J / \psi \gamma$ & +0.108 & +0.113 & -0.113 & -0.108 \\
$\chi_{c 2}(2 P) \rightarrow J / \psi \gamma$ & -0.143 & -0.151 & -0.203 & -0.192 \\
$\chi_{c 1}(2 P) \rightarrow \psi(2 S) \gamma$ & +0.034 & +0.036 & -0.036 & -0.034 \\
$\chi_{c 2}(2 P) \rightarrow \psi(2 S) \gamma$ & -0.041 & -0.043 & -0.058 & -0.055 \\
$\chi_{c 1}(3 P) \rightarrow J / \psi \gamma$ & +0.147 & +0.144 & -0.144 & -0.147 \\
$\chi_{c 2}(3 P) \rightarrow J / \psi \gamma$ & -0.213 & -0.207 & -0.277 & -0.286 \\
$\chi_{c 1}(3 P) \rightarrow \psi(2 S) \gamma$ & +0.086 & +0.078 & -0.078 & -0.086 \\
$\chi_{c 2}(3 P) \rightarrow \psi(2 S) \gamma$ & -0.107 & -0.096 & -0.128 & -0.144 \\
$\chi_{c 1}(3 P) \rightarrow \psi(3 S) \gamma$ & +0.038 & +0.026 & -0.026 & -0.038 \\
$\chi_{c 2}(3 P) \rightarrow \psi(3 S) \gamma$ & -0.046 & -0.031 & -0.041 & -0.062 \\
\hline \hline
\end{tabular}

